Kardiologe 2020 $14: 267-293$

https://doi.org/10.1007/s12181-020-00395-z

Online publiziert: 13. Mai 2020

(c) Deutsche Gesellschaft für Kardiologie Herz- und Kreislaufforschung e.V. Published by Springer Medizin Verlag $\mathrm{GmbH}$, ein Teil von Springer Nature - all rights reserved 2020

Tienush Rassaf' • Matthias Totzeck' - Johannes Backs ${ }^{2} \cdot$ Carsten Bokemeyer $^{3}$. Michael Hallek ${ }^{4}$. Denise Hilfiker-Kleiner ${ }^{5} \cdot$ Andreas Hochhaus $^{6}$. Diana Lüftner ${ }^{7}$. Oliver J. Müller ${ }^{8} \cdot$ Ulrich Neudorf $^{9} \cdot$ Roman Pfister $^{10} \cdot$ Stephan von Haehling ${ }^{11}$. Lorenz H. Lehmann ${ }^{12}$. Johann Bauersachs ${ }^{5}$ für die Kommission für Klinische Kardiovaskuläre Medizin der DGK

'Klinik für Kardiologie und Angiologie, Westdeutsches Herz- und Gefäßzentrum, Universitätsklinikum Essen, Essen, Deutschland; ${ }^{2}$ Institut für Experimentelle Kardiologie, Universitätsklinikum Heidelberg, Heidelberg, Deutschland; ${ }^{3}$ II. Medizinische Klinik (Onkologie, Hämatologie, Knochenmarktransplantation mit Abteilung für Pneumologie), Zentrum für Onkologie, Universitätsklinikum Hamburg-Eppendorf, Hamburg, Deutschland; ${ }^{4}$ Klinik I für Innere Medizin, Centrum für Integrierte Onkologie (CIO) $A B C D$, Universitätsklinikum Köln, Köln, Deutschland; ${ }^{5}$ Klinik für Kardiologie und Angiologie, Medizinische Hochschule Hannover, Hannover, Deutschland; ${ }^{6}$ Klinik für Innere Medizin II, Abteilung Hämatologie und Internistische Onkologie, Universitätsklinikum Jena, Jena, Deutschland; ' ${ }^{7}$ Klinik für Hämatologie, Onkologie und Tumorimmunologie, Charité Universitätsmedizin Berlin, Humboldt-Universität, Berlin, Deutschland; ${ }^{8}$ Klinik für Innere Medizin III mit den Schwerpunkten Kardiologie, Angiologie und internistische Intensivmedizin, Universitätsklinikum Schleswig-Holstein, Universität Kiel, Kiel, Deutschland; ${ }^{9}$ Klinik für Kinderheilkunde III, Westdeutsches Herz- und Gefäßzentrum Essen, Universitätsklinikum Essen, Essen, Deutschland; ${ }^{10} \mathrm{Klinik}$ III für Innere Medizin - Allgemeine und interventionelle Kardiologie, Elektrophysiologie, Angiologie, Pneumologie und internistische Intensivmedizin, Universitätsklinikum Köln, Köln, Deutschland; "Klinik für Kardiologie und Pneumologie, Herzzentrum Göttingen, Universitätsmedizin Göttingen, und Deutsches Zentrum für Herz- und Kreislaufforschung Standort Göttingen, Göttingen, Deutschland; ${ }^{12}$ Klinik für Kardiologie, Angiologie, Pneumologie, Sektion Kardio-Onkologie, Universitätsklinikum Heidelberg, Heidelberg, Deutschland

\title{
Onkologische Kardiologie
}

\section{Konsensuspapier der Deutschen Gesellschaft für Kardiologie - Herz- und Kreislaufforschung, der Deutschen Gesellschaft für Pädiatrische Kardiologie und Angeborene Herzfehler und der Deutschen Gesellschaft für Hämatologie und Medizinische Onkologie}

\section{Kardiovaskuläre Risikofaktoren und Erkrankungen - Risikoassessment vor Tumortherapie}

Aus Gründen der besseren Lesbarkeit und Verständlichkeit der Texte wird in SpringerPublikationen in der Regel das generische Maskulinum als geschlechtsneutrale Form verwendet. Diese Form impliziert immer alle Geschlechter.

Dieses Konsensuspapier wird simultan in deutscher Sprache in Der Kardiologe (https:// doi.org/10.1007/s12181-020-00395-z) und in englischer Sprache in Clinical Research in Cardiology (https://doi.org/10.1007/s00392020-01636-7) publiziert.

Lorenz Lehmann und Johann Bauersachs haben zu gleichen Teilen zum Manuskript beigetragen.
Viele onkologische Patienten haben vorbestehende Risikofaktoren oder kardiovaskuläre Erkrankungen, die durch die Krebserkrankung und insbesondere deren Behandlung zu einem weiten Spektrum an Komplikationen im Herz-Kreislauf-System führen können. Daneben wurde auch die Rolle einer genetischen Prädisposition für das Auftreten von Nebenwirkungen erkannt sowie der Einfluss weiterer von der Krebserkrankung selbst ausgehender und bisher unzureichend charakterisierter Faktoren (• Abb. 1; [11, 57, 99, 149]). Kardiovaskuläre Folgen reichen von diskreten elektrokardiographischen, laborchemischen oder in der Bildgebung aufgefallenen Veränderungen über das Auftreten von thrombembolischen, ischämischen oder rhythmologischen Ereignissen bis hin zur linksventrikulären (LV) Funktionseinschränkung und manifesten Herzinsuffizienz [140, 149]. Daher ist die Erfassung von kardiovaskulären Begleiterkrankungen durch die sorgfältige Erhebung der Anamnese sowie der Ausgangsbefunde vor onkologischen Therapieverfahren unverzichtbar. Neben der körperlichen Untersuchung 


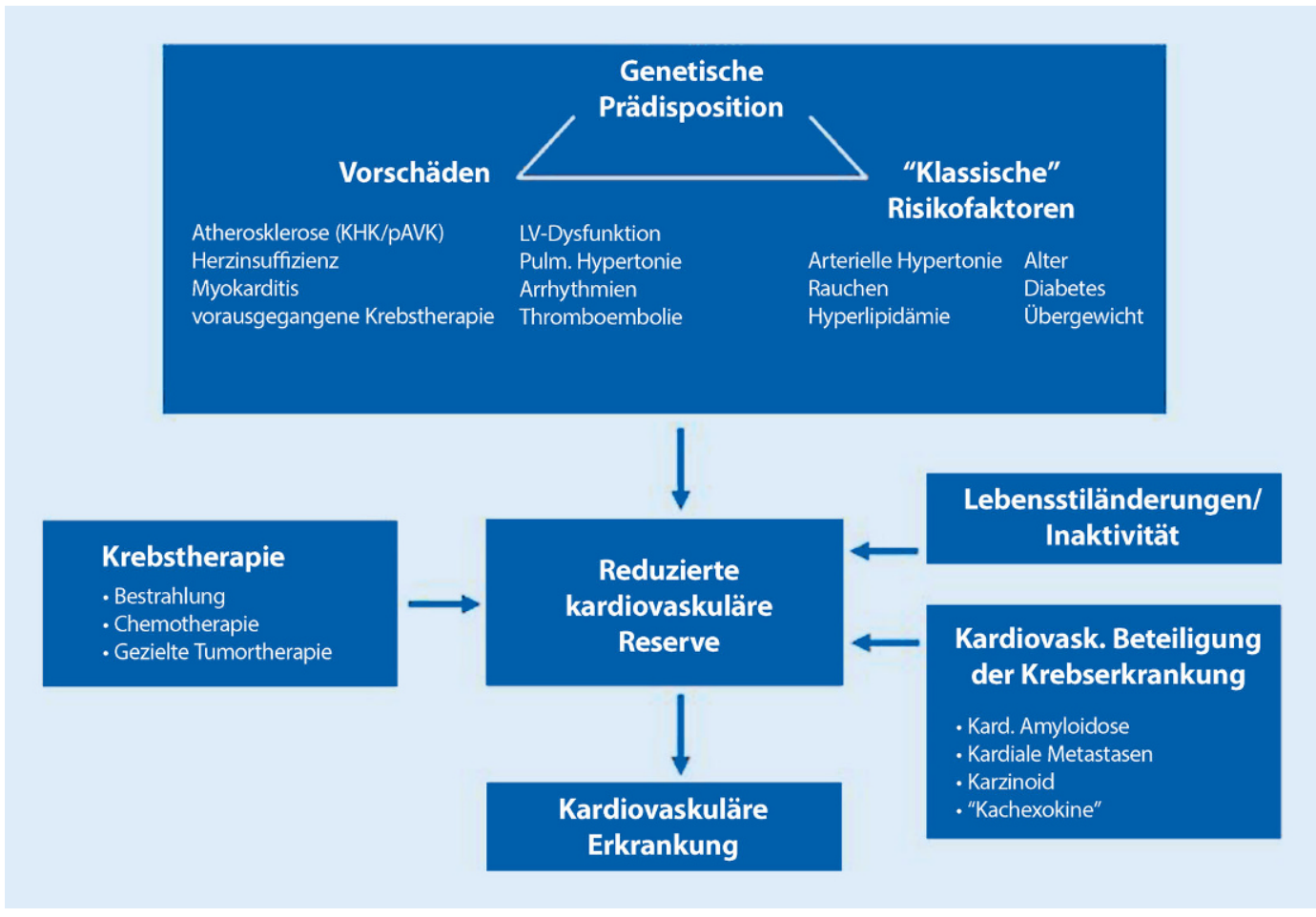

Abb. $1 \triangleleft$ Zusammenspiel von genetischer Prädisposition, klassischen Risikofaktoren und kardiovaskulären Vorerkrankungen für die Entwicklung oder Progression einer kardiovaskulären Erkrankung als Folge einer Krebstherapie („2nd Hit") oder der Krebserkrankung selbst. KHK koronare Herzerkrankung, $p A V K$ periphere arterielle Verschlusskrankheit, $L V$ linksventrikulär

spielen das Elektrokardiogramm (EKG) und Echokardiogramm einschließlich qualitativer und quantitativer Einschätzung der LV-Ejektionsfraktion (LVEF) eine zentrale Rolle, zunehmend auch die kardiale Magnetresonanztomographie (MRT). Bei subklinischen oder manifesten Auffälligkeiten kann ggf. eine alternative, weniger kardiotoxische Therapie gewählt werden, oder engmaschige Kontrollen erfolgen zur frühzeitigen Erkennung von Veränderungen im Herz-Kreislauf-System unter der Krebstherapie, was beispielsweise eine kardioprotektive Therapie ermöglicht.

Im Folgenden soll auf die Bedeutung klassischer Risikofaktoren, kardiovaskulärer Vor- bzw. Begleiterkrankungen sowie vorausgegangener Krebstherapien eingegangen werden.

\section{Klassische Risikofaktoren}

Kardiovaskuläre Erkrankungen und Krebserkrankungen teilen sich nicht nur gemeinsame Mechanismen wie chronische Inflammation, sondern auch gemeinsame Risikofaktoren [47, 90]. So gelten Diabetes mellitus, Adipositas und Hypercholesterinämie als Risikofaktoren für Brustkrebs und sind mit vermehr- ten kardiovaskulären Nebenwirkungen assoziiert [67, 101]. Auch wenn die in einer retrospektiven Studie bei Brustkrebspatientinnen gezeigte reduzierte Kardiotoxizität unter Statintherapie der Bestätigung in prospektiven Studien bedarf [17], halten wir die Behandlung einer Hypercholesterinämie für sinnvoll. Analog sollte auch ein Diabetes mellitus leitliniengerecht behandelt werden. Metformin sollte möglichst Teil der Therapie sein, da epidemiologische Studien eine reduzierte Krebsrate bei Diabetikern unter Metformin-Therapie nahelegen [44]. Rauchen als klassischer Risikofaktor von Krebserkrankungen ist ebenso mit vermehrter Atherosklerose bzw. koronarer Herzerkrankung (KHK) assoziiert [90] und sollte besonderes Augenmerk erfahren. Allerdings bleibt auch nicht abschließend geklärt, welche Bedeutung eine bestehende KHK für akute koronare Ereignisse unter Substanzen wie 5-Fluorouracil (5-FU) besitzt [1, 121].

Weniger bekannt ist, dass auch Bluthochdruck mit einer erhöhten Krebsrate zumindest bei Männern einhergeht und in beiden Geschlechtern mit einer gesteigerten Mortalität an Krebs [132]. Interessanterweise waren Inhibitoren des Renin-Angiotensin-Aldosteron-Sys- tems bei Krebserkrankten mit geringerer Metastasierung und verbessertem Überleben assoziiert [135]. Bereits vor Beginn einer Krebstherapie wie auch im Verlauf sollte daher eine arterielle Hypertonie identifiziert und behandelt werden.

Die Bedeutung der gemeinsamen Risikofaktoren zeigt sich auch bei Patienten mit geplanter Anthrazyklin-Therapie, bei denen ein erhöhtes Risiko zur Entwicklung einer Herzinsuffizienz bei vorbestehenden Risikofaktoren wie Rauchen, arterieller Hypertonie, Diabetes mellitus und Dyslipoproteinämie besteht [5]. Bei mehr als 2 Risikofaktoren scheint das Risiko deutlich erhöht zu sein [5-7]. Ein gesteigertes kardiovaskuläres Risiko unter klassischen kardialen Risikofaktoren besteht auch für Patienten mit Stammzelltransplantation [24] oder unter bestimmten Tyrosinkinaseinhibitoren wie Ponatinib oder Nilotinib, für die Hinweise auf den prognostischen Nutzen einer Risikofaktorenkontrolle bestehen [18].

\section{Kardiovaskuläre Vor- bzw. Begleiterkrankungen}

Die Identifikation kardiovaskulärer Vorund Begleiterkrankungen ist zentral für die Risikoeinschätzung. Neben der 
Anamnese hilft die körperliche Untersuchung, nicht nur eine Herzinsuffizienz $\mathrm{zu}$ identifizieren, sondern auch atherosklerotisch bedingte Veränderungen wie eine periphere arterielle Verschlusserkrankung, die z.B. durch manche Kinaseinhibitoren verkompliziert werden kann [88].

Wichtiger Bestandteil der kardialen Risikoerfassung ist das Erkennen von EKG-Veränderungen sowie von aktuellen bzw. früheren Herzrhythmusstörungen. Im 12-Kanal-EKG können bestimmte Medikamente potenziell gefährliche Verlängerungen der frequenzkorrigierten QT-Zeit (QTc) hervorrufen. Zwar stellen potenziell lebensbedrohliche Arrhythmien mit vorangehender QTc-Intervall-Verlängerung (u. a. Torsade de pointes [TdP]) auch in Risikokonstellationen seltene Ereignisse dar, es ist dennoch sinnvoll, vor einem Einsatz dieser QTc verlängernden Substanzen einen QTc-Ausgangswert zu erheben. Im Falle eines familiären Long-QT-Syndroms (LQTS) sollte von QTc verlängernden Substanzen Abstand genommen werden. Außerhalb von familiärem LQTS sind bestehende QTc-Verlängerungen durch eine Vormedikation insbesondere dann problematisch, wenn sie neben der bei Kinaseinhibitoren häufigen QTcVerlängerung auch zu dokumentiertem Auftreten von TdP führen können wie ggf. z. B. bei Vandetanib. Allerdings ist sicherlich eine Abwägung der Gesamtrisikokonstellation des Patienten sowie individueller QTc-Verlängerungen notwendig (www.crediblemeds.org) [89].

Im EKG können darüber hinaus Hinweise auf eine Myokardischämie gefunden werden, die insbesondere im Hinblick auf eine kardiale Ischämie auslösende Medikamente wie 5-FU eine weitere kardiale Diagnostik nahelegen. Zudem ist bei aktuellen oder anamnestischen Hinweisen auf Vorhofflimmern eine Antikoagulation in vielen Fällen indiziert, welche wiederum durch ein erhöhtes Blutungsrisiko eine Krebstherapie komplizieren kann. Umgekehrt sollte eine Antikoagulation bei Vorhofflimmern nicht unnötig vorenthalten werden [80]. Im Gegensatz zur venösen Thromboembolie konnte ein Zusammenhang zwischen Krebserkrankung und erhöhtem zerebra-

Kardiologe 2020 · 14:267-293 https://doi.org/10.1007/s12181-020-00395-z (c) Deutsche Gesellschaft für Kardiologie - Herz- und Kreislaufforschung e.V. Published by Springer Medizin Verlag $\mathrm{GmbH}$, ein Teil von Springer Nature - all rights reserved 2020

T. Rassaf · M. Totzeck · J. Backs - C. Bokemeyer - M. Hallek · D. Hilfiker-Kleiner · A. Hochhaus · D. Lüftner · O. J. Müller · U. Neudorf · R. Pfister · S. von Haehling · L. H. Lehmann · J. Bauersachs für die Kommission für Klinische Kardiovaskuläre Medizin der DGK

Onkologische Kardiologie. Konsensuspapier der Deutschen Gesellschaft für Kardiologie - Herz- und Kreislaufforschung, der Deutschen Gesellschaft für Pädiatrische Kardiologie und Angeborene Herzfehler und der Deutschen Gesellschaft für Hämatologie und Medizinische Onkologie

\section{Zusammenfassung}

Akut- und Langzeitnebenwirkungen der modernen, multimodalen Tumortherapie beeinträchtigen signifikant Lebensqualität und Überleben von Patienten mit Malignomen. Zu den wesentlichen Komponenten dieser Therapie zählen Strahlen-, die klassische (konventionelle) Chemo-, Immunsowie die sog. zielgerichteten Therapien. Zu etablierten Tumortherapiestrategien kommen bis zu 30 Neuzulassungen pro Jahr mit nur unvollständig charakterisierten Nebenwirkungsprofilen. Dieses Konsensuspapier adressiert die Risikofaktoren für eine mögliche adverse Reaktion unter Tumortherapie und führt im Weiteren spezifische Nebenwirkungsprofile für die verschiedenen Therapiegruppen auf. Schwerpunktmäßig wird auf neuartige Therapeutika eingegangen und Empfehlungen zur Behandlung spezifischer Patientengruppen aufgeführt.

\section{Schlüsselwörter}

Onkologische Kardiologie · Kardiotoxizität · Survivorship Programme $\cdot$ Krebstherapie . Chemotherapie

\section{Onco-Cardiology. Consensus Paper of the German Cardiac Society, the German Society for Pediatric Cardiology and Congenital Heart Defects and the German Society for Hematology and Medical Oncology}

\section{Abstract}

Acute and chronic side effects of modern, multimodal treatment of advanced cancers significantly impact the quality of life and survival in patients with malignant diseases. The essential components of these treatment modalities include radiotherapy, classical (conventional) chemotherapy, immunotherapy and the so-called molecular targeted therapies. In addition to the established treatment strategies up to 30 newly approved drugs enter the market each year, with only incompletely characterized side effect profiles. This consensus paper addresses the risk factors for potential adverse reactions under tumor treatment and furthermore lists specific side effect profiles for the various treatment groups. Novel cancer therapies are discussed and recommendations for the treatment of specific patient groups are presented.

Keywords

Cardio-oncology · Cardiotoxicity - Survivorship programs - Cancer therapy · Chemotherapy lem Ischämierisiko bei Vorhofflimmern nicht sicher nachgewiesen werden.

Bei Risikopatienten für kardiovaskuläre Komplikationen sollte eine Echokardiographie durchgeführt werden, da sie nicht nur eine subklinisch eingeschränkte LV-Pumpfunktion erfassen kann, sondern auch eine Beurteilung des Perikards, der Klappen und eine Abschätzung des pulmonalarteriellen Druckes als Ausgangswert für Verlaufs- kontrollen ermöglicht. Unklar ist der prädiktive Wert einer eingeschränkten LVEF für Stammzelltransplantationen. Bei geplanter Stammzelltransplantation geht die LVEF $<50 \%$ nur mit 1 Punkt in den sogenannten Sorror-Score zur Risikostratifizierung von Patienten ein [127, 128]. Eine leicht- bis mittelgradig eingeschränkte LVEF scheint kein Ausschlusskriterium für eine Stammzelltransplantation $\mathrm{zu}$ sein [112]. 
Bei anderen onkologischen Erkrankungen/Therapien, nimmt die eingeschränkte LVEF aber einen prädiktiven Wert ein [26]. Dies gilt auch für die LVEF vor Behandlung mit HER2-Inhibitoren/Antikörpern (nach erfolgter Anthrazyklin-Therapie) [50]. Aufgrund der aktuellen Empfehlung der American Society of Clinical Oncology (ASCO) sowie der Europäischen Gesellschaft für Kardiologie (European Society of Cardiology, ESC) ist daher ein Patient mit einer LVEF von kleiner 50-55\% als Risikopatient einzustufen [5, 149].

\section{Kardiotoxisches Risiko durch onkologische Vorbehandlung}

Viele onkologische Patienten durchlaufen mehrere potenziell kardiotoxische Therapien. Dies kann im Rahmen ihrer zugrunde liegenden onkologischen Erkrankung erfolgen (Progress oder Wiederauftreten) oder bei einer onkologischen Zweiterkrankung. Die stattgehabten onkologischen Therapien sind daher bezüglich einer potenziellen kardiotoxischen onkologischen Therapie als unabhängiger Risikofaktor anzusehen. Dies gilt insbesondere für Anthrazykline bzw. eine stattgehabte Bestrahlung [4]. Eine intensivierte Nachbetreuung onkologischer Patienten ist insbesondere bei einer Bestrahlung von >30 Gy, einer Anthrazyklin-Therapie mit mehr als $250 \mathrm{mg} / \mathrm{m}^{2}$ Körperoberfläche oder nach einer Kombinationstherapie notwendig. Somit zählen Patienten vor einer erneuten geplanten Radio- oder Chemotherapie zu einem Risikokollektiv und bedürfen einer genauen kardialen Abklärung und Betreuung.

Es ist zu erwarten, dass die Bedeutung neuer bildgebender Verfahren („strain rate" im Echokardiogramm, kontrastmittelfreies MRT, nuklearmedizinische Bildgebung) sowie genetischer Faktoren und Biomarker zunehmen wird. Die bisherigen Hinweise auf genetische Faktoren erstrecken sich überwiegend auf die Anthrazyklin-Toxizität $[11,57]$ und haben noch keinen Eingang in die klinische Routine gefunden. Auch die Bedeutung von tumorbedingten kardiodepressorischen Faktoren, die über die Änderungen des Lebensstils von Krebspatienten hinausgehen (z. B. Inaktivität), oder Risikofaktoren wie die klonale Hämatopoese sind noch unklar.

Schlussfolgerungen:

- Vor geplanter onkologischer Therapie sind kardiale Risikofaktoren sowie die Historie potenziell kardiotoxischer Therapien entscheidend für die individuelle Einschätzung.

Drei spezifische Patientengruppen bedürfen einer engen kardiologischen Mitbetreuung unter onkologischer Therapie:

- Patienten mit kardiovaskulären Risikofaktoren. Sie haben ein erhöhtes Risiko für kardiovaskuläre Nebenwirkungen einer Krebstherapie.

- Patienten mit einer stattgehabten systemischen Therapie bzw. Radiotherapie des Thorax gehören ebenfalls zu einem Hochrisikokollektiv für die Entwicklung einer Kardiotoxizität.

- Patienten mit einer geplanten Therapie mit einem deutlich erhöhten Risiko für eine Kardiotoxizität benötigen eine genaue kardiale Basisuntersuchung, um eine Verlaufsbeurteilung zu ermöglichen.

\section{Strahlentherapie}

Die Strahlentherapie ist ein wichtiger Bestandteil eines multimodalen Therapiekonzepts vieler Krebserkrankungen. Sie wird zur lokalen Tumorkontrolle und als Therapie von Metastasen mit palliativer Zielsetzung sowie mit kurativer Zielsetzung als neoadjuvante, adjuvante oder als definitive Therapie bei lokalisierten Krebserkrankungen eingesetzt. Bei 35\% aller Patienten mit Krebserkrankung erfolgt innerhalb eines Jahres nach Diagnosestellung eine Strahlentherapie [87, 141]. Eine kardiale Strahlenexposition kann v. a. durch Bestrahlung eines mediastinalen Lymphoms, eines zentral lokalisierten Bronchialkarzinoms oder eines linksseitigen Mammakarzinoms erfolgen [9, 141, 149].

Strahlentherapieassoziierte, kardiovaskuläre Komplikationen können sich akut, jedoch auch chronisch im Verlauf von über 20 Jahren nach Strahlenexposition manifestieren. Die strahlen- therapieassoziierte Perikarditis ist eine akute Komplikation nach Strahlentherapie [141]. Die akute Verlaufsform galt als die häufigste kardiale Komplikation nach Strahlentherapie, jedoch hat die Inzidenz durch eine Verminderung der kardialen Einzeldosis und durch technische Verbesserungen abgenommen [49]. Sie ist charakterisiert durch eine ausgeprägte perikardiale Immunzellinfiltration und zeigt häufig eine exsudative Verlaufsform. Die chronische, strahlentherapieassoziierte Perikarditis kann sich durch variables Auftreten zeigen und tritt erst verzögert nach Strahlentherapie auf. Ungefähr $20 \%$ der Patienten mit einer chronischen Perikarditis entwickeln eine klinisch-relevante Konstriktion des Herzens. Die kumulative kardiale Strahlendosis gilt als Hauptrisikofaktor; bei einer Dosis von 40 Gy liegt die Inzidenz einer manifesten Perikarditis bei $5 \%$ innerhalb von 5 Jahren nach Strahlentherapie [29, 141]. Nach Strahlenchemotherapie konnte in $31,4 \%$ ein Perikarderguss innerhalb eines Jahres nachgewiesen werden. Die Diagnostik umfasst vor allem Echokardiographie und Herzkatheteruntersuchung. Bei konstriktiver Perikarditis ist die komplette Perikardektomie Therapie der Wahl [141].

Die KHK, kalzifizierende Herzklappenerkrankungen und myokardiale $\mathrm{Fi}$ brose mit diastolischer sowie seltener systolischer Dysfunktion sind typische chronische, strahlentherapieassoziierte Komplikationen [141, 149]. Risikofaktoren sind die kumulative Strahlendosis, eine begleitende Anthrazyklin-Chemotherapie, ein hohes kardiovaskuläres Risikoprofil und bestehende kardiovaskuläre Vorerkrankungen [58, 60, 141, 149]. Patienten nach überlebter Krebserkrankung in der Kindheit stellen eine besondere Risikogruppe dar. Das relative Risiko für eine schwere kardiale Erkrankung im Alter von 40 Jahren liegt bei einer kardialen Strahlendosis von $1-5$ Gy bei 1,9 und steigt bei $>15$ Gy auf 19,5-75,2 [53].

Die KHK ist die häufigste strahlentherapieassoziierte, kardiovaskuläre Komplikation nach Mammakarzinom. Ein erhöhtes Risiko für die Entwicklung einer KHK besteht zudem nach mehr als 20 Jahren nach Bestrahlung [32, 138]. 
Hier steht eine Anzeige.

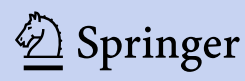




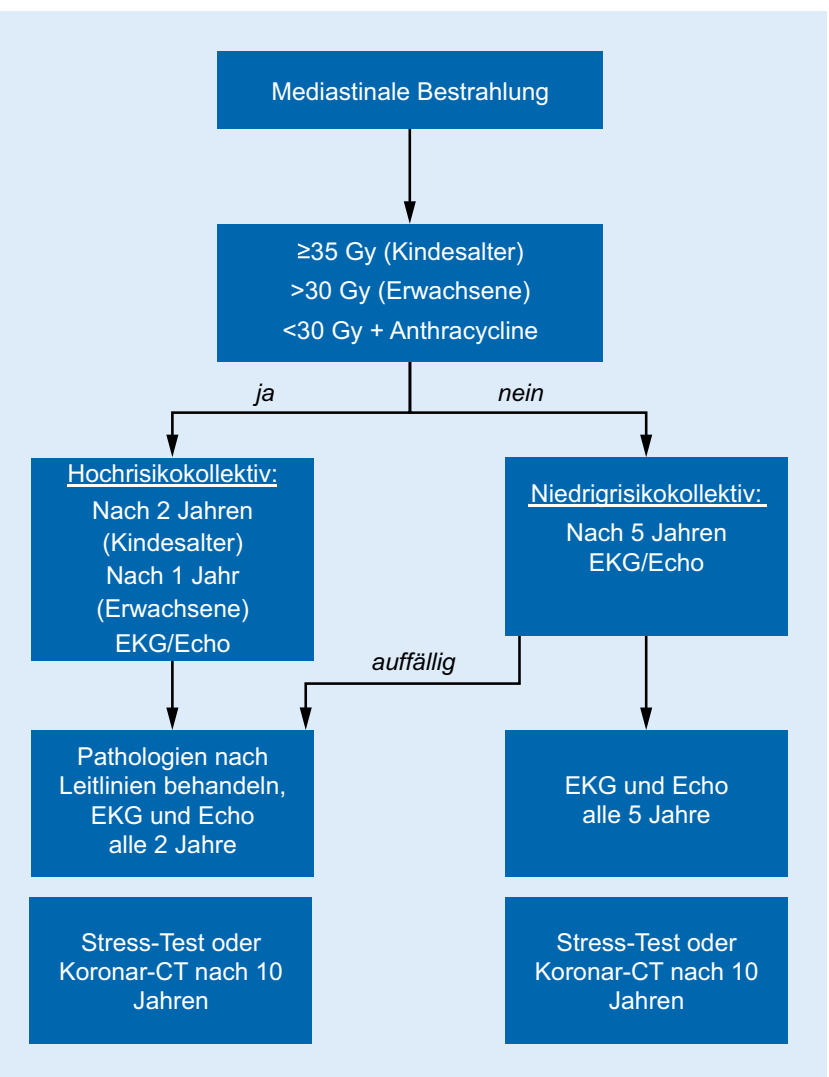

Das individuelle Risiko nimmt pro $1 \mathrm{~Gy}$ kardialer Strahlendosis um 4,1-7,4\% zu [32, 138, 141]. Betroffene Koronararterien sind abhängig von charakteristischen Strahlenfeldern; die Bestrahlung eines linksseitigen Mammakarzinoms ist assoziiert mit einer Strahlenexposition des Ramus interventricularis anterior (RIVA), wohingegen die mediastinale Bestrahlung zu einer Strahlenexposition des linken Hauptstamms sowie des Ramus circumflexus (RCX) und der rechten Koronararterie (RCA) führt. Es zeigt sich zudem insgesamt ein gehäuftes Auftreten von ostialen Läsionen nach Strahlentherapie [149].

Die mediastinale Bestrahlung beim Hodgkin-Lymphom ist charakterisiert durch eine höhere Strahlenexposition der Herzklappenebene und ein erhöhtes Risiko für Herzklappenerkrankungen, die Latenz liegt bei über 20 Jahren [138, 141]. Insbesondere bei einer kumulativen Strahlendosis von >30 Gy nimmt das relative Risiko exponentiell $\mathrm{zu}$ und beträgt bei $>40$ Gy 24,3\% [30, 138]. Bei Langzeitüberlebenden nach thorakaler Bestrahlung mit oder ohne begleitende
Abb. $2<$ Algorithmus zum kardiologischen Follow-up nach mediastinaler Bestrahlung. Gy Gray, EKG Elektrokardiogramm, Echo Echokardiographie, CTComputertomograhie diovaskulären Schädigung sind daher von besonderer Relevanz.

Bereits vor Einleitung einer thorakalen Strahlentherapie sollte bei Patienten mit erhöhtem kardiovaskulärem Risikoprofil oder begleitender, kardiotoxischer Chemotherapie (insbesondere Anthrazykline) eine Risikobeurteilung erfolgen. So können vor Einleitung einer Strahlentherapie eine weiterführende Diagnostik sowie eine interdisziplinäre, risikoadaptierte Therapieplanung erfolgen [144].

Zur Überwachung von strahlentherapieassoziierten, kardiovaskulären Komplikationen sollte ab 5 Jahren nach Strahlenexposition in einem Intervall von 2 bis 5 Jahren in Abhängigkeit des individuellen Risikos eine onkokardiologische Verlaufsbeurteilung inklusive EKG und Echokardiographie erfolgen $[21,73,141]$. Nach Bestrahlung im Kindesalter von $\geq 35 \mathrm{~Gy}$, einer Strahlendosis $>30$ Gy oder begleitender AnthrazyklinTherapie sollte bereits frühzeitig (Kinder 2 Jahre, Erwachsene 1 Jahr nach Bestrahlung) eine kardiale Beurteilung erfolgen (• Abb. 2; [4]). Die Basisdiagnostik kann durch eine Strain-Analyse oder eine kardiale MRT bei eingeschränkter Qualität der Echokardiographie ergänzt werden [8]. Eine nichtinvasive KHK-Diagnostik kann 10 Jahre nach Strahlenexposition mittels Koronar-CT (Computertomographie) oder Evaluation einer belastungsinduzierbaren, myokardialen Ischämie erfolgen [21].

\section{Klassische Chemotherapie}

\section{Management von Patienten nach Bestrahlungstherapie}

Die Diagnose und Behandlung von kardiovaskulären Komplikationen nach Strahlentherapie ist durch verschiedene Faktoren erschwert; eine strahlentherapieassoziierte KHK kann durch eine strahlungsbedingte Neuropathie eine atypische oder verminderte Symptomatik zeigen [73]. Aufgrund proximaler Läsionen, mediastinaler Verwachsungen und der zugrunde liegenden Tumorerkrankung ist die Komplikationsrate einer interventionellen und insbesondere einer operativen Versorgung erhöht. Die frühzeitige Diagnostik und Therapie einer strahlentherapieassoziierten kar-

Die klassische, zytotoxische Chemotherapie kann zu einem breiten Spektrum von kardiovaskulären Nebenwirkungen führen. Insbesondere die Gruppe der Anthrazykline (Doxorubicin, Epirubicin, Daunorubicin, Idarubicin, Mitoxantron) ist mit einem erheblichen Risiko für eine myokardiale Schädigung vergesellschaftet. Anthrazykline sind weit verbreitet für die Therapie akuter Leukämien, des Mammakarzinoms und des malignen Lymphoms. Eine myokardiale Schädigung wird durch reaktive Sauerstoffspezies (ROS), Hemmung der Topoisomerase II $\beta$ und eine Wechselwirkung mit Kalziumkanälen verursacht. Diese Veränderungen kön- 
nen eine LV-Dysfunktion verursachen, welche zu einer manifesten Herzinsuffizienz als unabhängigem Prädiktor einer gesteigerten Mortalität führen kann. Das Risiko einer Anthrazyklin-assoziierten Kardiotoxizität ist abhängig von der kumulativen Dosis, der Höchstmenge von Einzeldosen sowie den Risikofaktoren des Patienten und wird gesteigert durch eine begleitende thorakale Bestrahlung oder HER2-Inhibitortherapie [141]. Das Risiko für eine LV-Dysfunktion liegt bei einer Therapie mit Doxorubicin $400 \mathrm{mg} / \mathrm{m}^{2}$ Kumulativdosis bei $5 \%$ und ist bei einer kumulativen Dosis von $550 \mathrm{mg} / \mathrm{m}^{2}$ bereits auf $26 \%$ gesteigert [149]. Am besten untersucht ist Doxorubicin als unverzichtbarer Standard in der Chemotherapie des Mammakarzinoms $\left(\mathrm{AC}=\right.$ Adriamycin $^{\mathrm{R}}+$ Cyclophosphamid $)$ sowie hochmaligner Lymphome (CHOP = Cyclophosphamid, Vincristin, Doxorubicin und Vincristin). Mit Einzeldosen von üblicherweise $50-60 \mathrm{mg} / \mathrm{m}^{2}$ und 4 bis 6 Zyklen im Rahmen einer adjuvanten bzw. kurativen Chemotherapie wird Doxorubicin bis an die Grenzdosis von $400 \mathrm{mg} / \mathrm{m}^{2}$ ausgereizt. Bis zu diesem Limit gilt die 5- bis 10-Jahres-Inzidenz von Kardiomyopathien als geringfügig mit einer Wahrscheinlichkeit bis maximal 5\%. Bei Überschreitung dieser Grenzdosis steigt die Zahl von Patienten, die eine LV-Dysfunktion entwickeln exponentiell an, sodass Re-Expositionen vermieden und allenfalls auf liposomale Anthrazykline zurückgegriffen werden sollte.

Nach Beurteilung des individuellen Risikos für die Entstehung einer Anthrazyklin-assoziierten Kardiotoxizität erfolgt die Überwachung anhand klinischer Verlaufskontrollen, EKG und Echokardiographie ggf. mit 3-D-LVEF und Strain-Analyse [141]. Die Bestimmung des kardialen Troponins kurz nach Therapieapplikation hat eine gute prädiktive Wertigkeit [92]. Bei Patienten mit hohem Risiko für eine Anthrazyklinassoziierte Kardiotoxizität, Patienten mit einer Troponinerhöhung unter Therapie und Patienten mit bereits eingetretener LV-Dysfunktion kann die Therapie mit einem Angiotensin-Converting-Enzyme(ACE)-Inhibitor/Angiotensin-IIRezeptor-Subtyp-1(AT1)-Rezeptoranta- gonist und/oder einem $\beta$-Blocker zur Prävention oder Therapie eingesetzt werden. Zur Reduktion der Kardiotoxizität durch Anthrazykline eignen sich eine Therapie mit dem Eisenchelator Dexrazoxan, die Verabreichung einer alternativen Anthrazyklin-Formulierung (pegylierte und/oder liposomale Anthrazykline) oder die Umstellung auf ein nicht-Anthrazyklin-haltiges Therapiekonzept [21, 141].

Alkylanzien (Cyclophosphamid, Ifosfamid) werden für die Therapie von malignen hämatologischen Neoplasien, soliden Tumoren und Autoimmunerkrankungen eingesetzt, und sind insbesondere bei hoher Dosierung vor Knochenmarktransplantation mit kardiovaskulären Komplikationen vergesellschaftet. Eine Cyclophosphamidassoziierte Herzinsuffizienz (Inzidenz 5-19\%) entsteht typischerweise binnen weniger Tage nach Therapieapplikation. Als Pathomechanismus werden eine Lipidperoxidation, eine mitochondriale Dysfunktion sowie die Generierung von ROS vermutet. Eine präventive Therapie mit ACE-Inhibitoren/AT1-Rezeptorantagonisten und/oder einem $\beta$-Blocker wurde für Alkylanzien bisher nicht ausreichend untersucht. Cyclophosphamid wird in Dosierungen zwischen 600 und $2000 \mathrm{mg} / \mathrm{m}^{2}$ eingesetzt und gilt als relativ unproblematisch, bei höheren Dosen kann es zur oben genannten kardialen Schädigung kommen.

Fluoropyrimidine (5-FU) können durch eine Vasospasmus induzierende Wirkung an Koronararterien $\mathrm{zu}$ transienter Angina pectoris bis hin zum akuten Myokardinfarkt führen. Ein Vasospasmus tritt typischerweise in der frühen Therapiephase auf. Das Risiko ist abhängig von der Applikationsart, der Dosis sowie der individuellen Prädisposition und kann bis $\mathrm{zu} 10 \%$ betragen. Kalziumantagonisten und Nitrate wurden erfolgreich für die Therapie eines koronararteriellen Vasospasmus durch Fluoropyrimidine angewendet [21, 92, 141]. Platinderivate (Cisplatin, Carboplatin), welche beispielsweise für die Therapie des kolorektalen Karzinoms und von Hodentumoren verwendet werden, können durch eine prokoagulatorische Wirkung und die Induktion einer endo- thelialen Dysfunktion durch arterielle Thrombosen/Thrombembolien ebenfalls eine myokardiale Ischämie verursachen. Darüber hinaus ist bei Patienten nach platinhaltiger Chemotherapie das Risiko für die Entwicklung einer KHK innerhalb von 20 Jahren gesteigert [149].

\section{Immuncheckpoint-Inhibitor- induzierte Myokarditis}

Immuncheckpoint-Inhibitoren (ICIs) wurden 2018 in mehr als 2250 aktiven klinischen Studien zur Behandlung maligner Erkrankungen eingesetzt. Diese neue Gruppe von Medikamenten ist häufig mit autoimmunvermittelten $\mathrm{Ne}$ benwirkungen assoziiert. Limitierend für eine weiterführende Therapie ist die ICIinduzierte Myokarditis [39, 85, 96, 97, 120]. Mit $43-46 \%$ hat die ICI-induzierte Myokarditis zudem eine hohe Mortalität und tritt je nach Medikament mit einer Häufigkeit von 1-2\% auf [85]. Unklar ist, wie Hochrisikopatienten identifiziert werden können [16].

\section{Klinische Symptomatik}

Die klinische Manifestation reicht von einem isolierten Anstieg kardialer Biomarker (Troponinanstieg, NT-proBNPAnstieg [N-terminal-pro hormone brain natriuretic peptide]) über Arrhythmien und sekundäre Anzeichen einer Herzinsuffizienz bis hin zu fatalen Ereignissen [85]. Typische oder atypische Angina pectoris tritt bei bis zu $37 \%$ der Patienten auf [85], eine myokardiale Ischämie ist die wichtigste Differenzialdiagnose.

\section{Hauptmerkmale der ICI- Myokarditis}

Die meisten Fälle (bis zu 81 \%) von ICIinduzierter Myokarditis treten in den ersten 3 Monaten nach Initialisierung der Behandlung auf [85]. Bisher sind nur wenige spezifische Risikofaktoren für eine ICI-induzierte Myokarditis bekannt; wichtigster unabhängiger Risikofaktor ist die Kombinationstherapie verschiedener ICIs (CTLA-4-Inhibitor [cytotoxic T-lymphocyte-associated Protein 4 inhibitor] + PD-1-Inhibitor [programmed cell death protein 1 inhibitor]) [85]. Pati- 


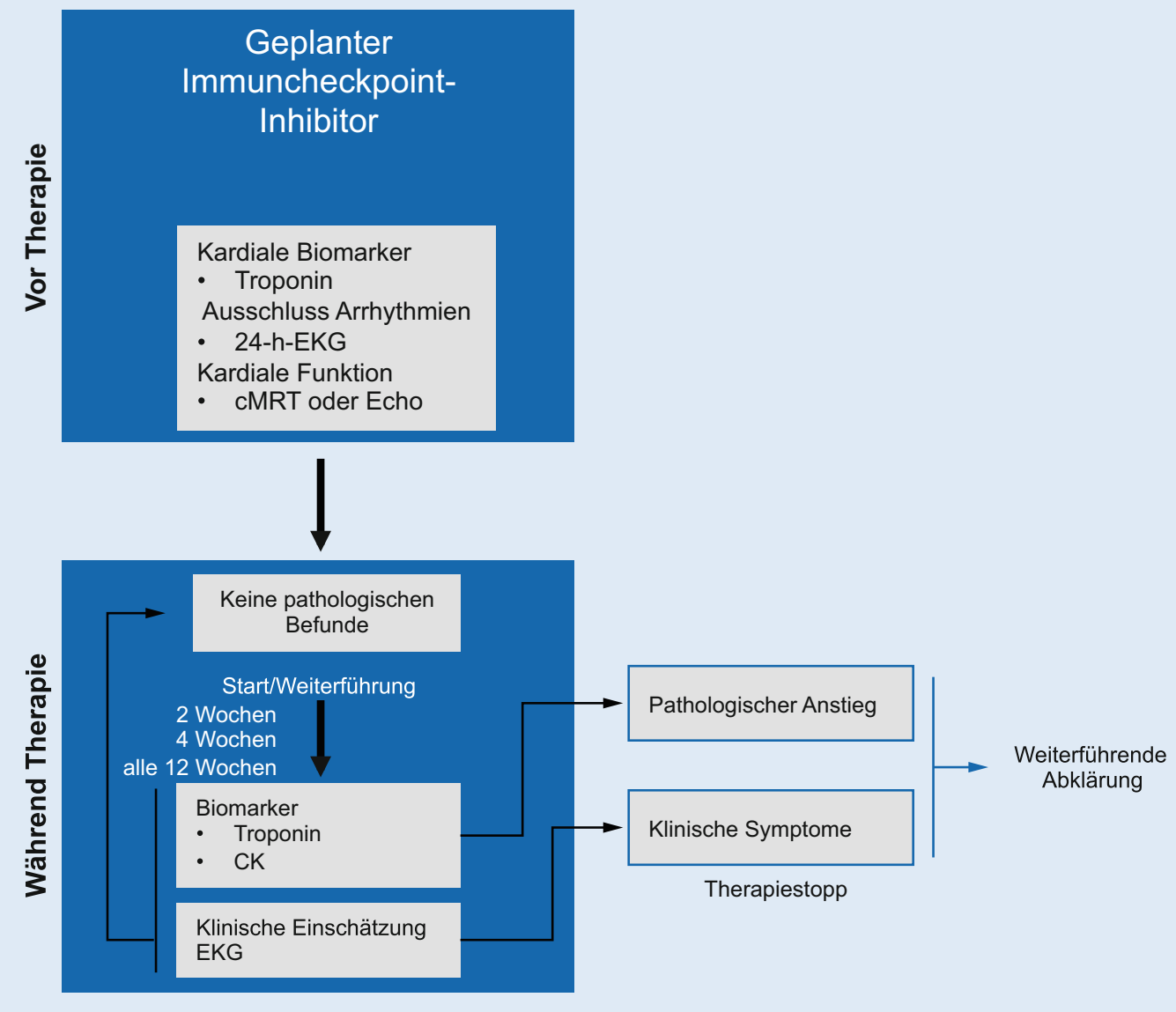

Abb. $3<$ Übersicht über die empfohlenen Basisund Verlaufsuntersuchungen bei einer Therapie mit einem Immuncheckpoint-Inhibitor. Die parallele Bestimmung von Troponin und Kreatininkinase (CK) ist aufgrund der oft begleitenden Myositis sinnvoll. EKG Elektrokardiogramm, CMRT kardiale Magnetresonanztomographie, Echo Echokardiographie

enten mit Thymom scheinen zudem ein erhöhtes Risiko für eine ICI-induzierte Myokarditis zu haben.

Myositis (23-30\%), eine Myasthenieähnliche Symptomatik, Diplopie (bis zu 6\%) oder eine Einschränkung der Zwerchfellfunktion treten bei Patienten mit ICI-induzierter Myokarditis auf [63, 85, 97, 120]. Bei Patienten mit ICI-assoziierter Myasthenie kann eine Myokarditis in 3-10\% [136] oder eine Myositis in 10-15\% nachgewiesen werden [3]. Patienten mit konkomitanten Myastheniesymptomen und Myokarditis haben eine hohe Sterblichkeitsrate [136].

\section{EKG-Veränderungen}

EKG-Veränderungen sind bei ICI-induzierter Myokarditis häufig. Sie reichen von ST-Hebungen über ventrikuläre Arrhythmien und Überleitungsstörungen bis hin zu kompletten Blockbildern. Ein abnormales EKG kann bei den meisten Patienten gefunden werden und scheint eines der sichersten Anzeichen einer ICIinduzierten Myokarditis zu sein [39, 85]. Arrhythmien sind ebenfalls stark mit ICIinduzierter Myokarditis assoziiert und oft ursächlich für fatale Verläufe [39, 64, 85, $119,120]$. Berichtet wurden Vorhofflattern, höhergradige AV-Blöcke, Kammerflimmern und Asystolie [96]. Bei Auftreten von EKG-Veränderungen unter ICI muss eine weiterführende Diagnostik inklusive kardialer Biomarker initiiert werden (• Abb. 3) und ein EKG-Monitoring erwogen werden.

\section{Biomarker}

Bei mehr als 90\% der Patienten mit ICI-induzierter Myokarditis liegt eine Troponin-Erhöhung vor [96]. Bisher gibt es keine eindeutige Grenze für die Diagnose einer ICI-induzierten Myokarditis, höhere Werte waren jedoch mit einer höheren Inzidenz tödlicher Ereignisse verbunden [85]. Basierend auf den ESC-Guidelines zur Diagnostik und Therapie des STEMI/NSTEMI (STElevationsmyokardinfarkt/Non-ST-Elevationsmyokardinfarkt) muss zunächst eine ischämische Genese bzw. eine andere mögliche Ursache für eine TroponinErhöhung ausgeschlossen werden [2]. NT-proBNP ist als Prognosemarker bei Patienten mit Herzinsuffizienz etabliert. Insbesondere bei Patienten mit stark verminderter LVEF und ICI-induzierter Myokarditis kann NT-proBNP als wertvoller Follow-up-Parameter dienen.

\section{Kardiale Bildgebung}

Eine verringerte LVEF ist nur bei ca. $50 \%$ der Patienten mit Myokarditis unter ICI zu beobachten [39, 85]. Eine reduzierte LVEF wirkt sich jedoch negativ auf das "Outcome“ aus und ist mit teils erheblichen Komplikationen verbunden. Um Veränderungen im Verlauf beurteilen zu können, sollte vor Einleitung einer ICITherapie eine Echokardiographie erfolgen. 
Tab. 1 Diagnostische Kriterien für die ICl-induzierte Myokarditis. (Nach Hu et al. [59])

\begin{tabular}{|c|c|c|c|c|}
\hline & Histopathologie & CMRT & Echokardiogramm mit neuen WBS & $\begin{array}{l}\text { Erhöhte kardiale Biomarker } \\
\text { im Vergleich zu Vorwerten }\end{array}$ \\
\hline Gesichert & $\begin{array}{l}\text { Pathologischer Be- } \\
\text { fund aussagekräftig }\end{array}$ & $\begin{array}{l}\text { cMRT } \\
+ \\
\text { M-Syndrom } \\
+ \\
\text { erhöhte kardiale Biomarker oder } \\
\text { EKG-Veränderungen }\end{array}$ & $\begin{array}{l}\text { WBS } \\
+ \\
\text { M-Syndrom } \\
+ \\
\text { erhöhte kardiale Biomarker } \\
+ \\
\text { EKG-Veränderungen } \\
+ \\
\text { Abwesenheit von stenosierender koro- } \\
\text { narer Herzerkrankung }\end{array}$ & - \\
\hline \multirow[t]{2}{*}{$\begin{array}{l}\text { Wahr- } \\
\text { schein- } \\
\text { lich }\end{array}$} & - & $\begin{array}{l}\text { cMRT } \\
\text { ohne M-Syndrom } \\
\text { ohne erhöhte kardiale Biomarker } \\
\text { ohne EKG-Veränderungen }\end{array}$ & $\begin{array}{l}\text { WBS } \\
+ \\
\text { M-Syndrom } \\
+ \\
\text { Erhöhte kardiale Biomarker } \\
\text { oder } \\
\text { EKG-Veränderungen }\end{array}$ & - \\
\hline & - & $\begin{array}{l}\text { Nicht eindeutiger cMRT-Befund } \\
+ \text { M-Syndrom } \\
\text { oder } \\
\text { erhöhte kardiale Biomarker } \\
\text { oder } \\
\text { EKG-Veränderungen }\end{array}$ & - & - \\
\hline Möglich & - & $\begin{array}{l}\text { Nicht eindeutiger cMRT-Befund } \\
\text { ohne M-Syndrom } \\
\text { ohne erhöhte kardiale Biomarker } \\
\text { ohne EKG-Veränderungen }\end{array}$ & $\begin{array}{l}\text { WBS } \\
+ \\
\text { M-Syndrom } \\
\text { oder } \\
\text { EKG-Veränderungen }\end{array}$ & $\begin{array}{l}\text { Erhöhte kardiale Biomarker } \\
+ \\
\text { M-Syndrom } \\
\text { oder } \\
\text { EKG-Veränderungen }\end{array}$ \\
\hline
\end{tabular}

Das kardiale MRT kann den Verdacht auf ICI-induzierte Myokarditis durch nicht-ischämisch bedingtes Late Gadolininum Enhancement (LGE) untermauern. LGE kann bei Patienten mit ICI-induzierter Myokarditis in 26-36\% nachgewiesen werden [85]. Neben LGE liefert das cMRT die LVEF und sollte nach Möglichkeit bei Patienten mit Verdacht auf ICI-induzierte Myokarditis zusätzlich zur Echokardiographie durchgeführt werden.

\section{Diagnostik bei Verdacht auf eine $\mathrm{ICl}$-induzierte Myokarditis}

Die Diagnostik umfasst standardmäßig kardiale Biomarker, ein EKG und eine Echokardiographie und bei fortbestehendem Verdacht ein kardiales MRT. Eine begleitende Myastheniesymptomatik sollte zudem evaluiert werden. Zudem muss eine signifikante Koronarobstruktion ausgeschlossen werden. Die Myokardbiopsie stellt den Goldstandard der Diagnostik einer Myokarditis dar und sollte im Verdachtsfall angestrebt werden. Im Falle der ICI ist eine lymphozytäre Infiltration typisch [64]. Zur weiteren Risikostratifizierung sollte ein 24h-EKG initialisiert werden [84]. Die aktuellen Kriterien zur Diagnostik sind in - Tab. 1 zusammengefasst.

\section{Ergänzende Diagnostik}

Anti-Acetylcholin-Rezeptor-Antikörper sollten bei Patienten mit Myastheniesyndrom bestimmt werden, um weitere therapeutische Strategien (z.B. Plasmapherese, Pyridostigmin-Therapie) in Betracht zu ziehen. Zusätzliche Untersuchungen (peripheres CD4/CD8-Verhältnis, 18F-Fluordesoxyglucose-Positronen-Emissions-Tomographie (FDGPET)/CT-Scan) können im individuellen Fall eine diagnostische Stütze bilden. Eine Skelettmuskelbiopsie sollte bei Patienten mit einem Anstieg der CK bzw. klinischen Anzeichen einer Myositis und Verdacht auf eine ICIinduzierte Myokarditis in Betracht gezo- gen werden, wenn keine Myokardbiopsie durchführbar ist.

Auch bei guter LVEF oder Vorliegen einer signifikanten KHK sollte bei Verdacht auf eine ICI-induzierte Myokarditis eine Myokardbiopsie in Betracht gezogen werden [39, 85]. Insbesondere Patienten mit klinischer Symptomatik, erhöhter CK, EKG-Veränderungen, malignen Arrhythmien, nicht typischem LGE im cMRT oder einer reduzierten LVEF, die durch eine KHK nicht ausreichend erklärt ist, benötigen eine Myokardbiopsie.

\section{Grundlagen der Therapie der ICI- induzierten Myokarditis}

Die eigentliche Therapie der ICI-induzierten Myokarditis beruht zunächst auf einer gewichtsadaptierten Steroidgabe sowie einer supportiven Therapie [59] mit 1-2 mg/kg Prednisolon i.v. oder oral bzw. 500-1000 mg Methylprednisolon i.v. bei schlechtem Ansprechen [13]. Ein Ausschleichen kann über 4 bis 6 Wochen erfolgen [84]. Bei fehlendem Anspre- 
Hier steht eine Anzeige.

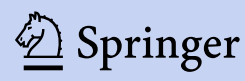


Hier steht eine Anzeige.

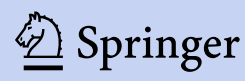


Tab. 2 Zielstruktur und Halbwertszeit der Immuncheckpoint-Inhibitoren. (Mod. nach [20])

\begin{tabular}{l|l|l} 
& Zielstruktur & Halbwertszeit (in d) \\
\hline Ipilimumab & CTLA-4 & 15 \\
\hline $\begin{array}{l}\text { Atezolizumab } \\
\text { Avelumab }\end{array}$ & PD-L1 & 27 \\
$\begin{array}{l}\text { Durvalumab } \\
\text { Nivolumab }\end{array}$ & 6,1 \\
$\begin{array}{l}\text { Pembrolizumab } \\
\text { Cemiplimab }\end{array}$ & 21 \\
\hline $\begin{array}{l}\text { CTLA-4 „cytotoxic t-lymphocyte-associated-protein 4", PD-L1 „programmed cell death-1 ligand 1", } \\
\text { PD-1 „programmed cell death-1" }\end{array}$ & 25 \\
\hline
\end{tabular}

chen, Auftreten von Komplikationen (höhergradige Arrhythmien, hämodynamische Instabilität oder Verschlechterung der LVEF) ist eine zusätzliche Immunsuppression bzw. eine Plasmapherese, auch aufgrund der teils langen Halbwertszeiten der Medikamente (• Tab. 2) anzuraten. Als zusätzliche immunsuppressive Therapie werden aktuell Tacrolimus, Infliximab oder Mycophenolat vorgeschlagen [59]. Spezielle Fällen mit einem hohen Risiko (hämodynamische Instabilität, vermehrtes Auftreten von Arrhythmien) könnten zudem von einer gezielten Antikörperbehandlung mit Abatacept, einem CTLA-4-Fusionsprotein, profitieren [119]. Aufgrund der mangelnden Studienlage muss in schweren Fällen eine individuelle Strategie etabliert werden. Diese Patienten sollten aufgrund ihrer hohen Mortalität unbedingt intensivmedizinisch versorgt werden. Bis zum sicheren Ausheilen der Myokarditis sollte keine weitere Therapie mit einem ICI erfolgen. Eine manifeste Herzinsuffizienz sollte analog den aktuellen Herzinsuffizienzleitlinien behandelt werden.

\section{Basisdiagnostik und Monitoring von Patienten mit ICl-Therapie}

Es gibt noch keine sichere Festlegung, welche Diagnostik vor Initialisierung einer ICI-Therapie zwingend erforderlich ist. Aufgrund der Schwere der möglichen Myokarditis empfehlen wir eine Bestimmung der kardialen Biomarker, die Durchführung einer Echokardiographie sowie ein EKG, um Vergleichsbefunde im Falle des Verdachts einer ICI-induzierten Myokarditis zu haben (• Abb. 3).
Während der Therapie empfiehlt sich neben der regelmäßigen Anamneseerhebung hinsichtlich kardialer Symptome ein EKG-Monitoring v. a. innerhalb der ersten 12 Wochen aufgrund des in diesem Zeitraum gehäuften Auftretens. Bei klinischer Symptomatik oder pathologischen Befunden sollte umgehend bei Verdacht auf eine ICI-induzierte Myokarditis eine weiterführende Diagnostik erfolgen.

\section{Zielgerichtete Therapien: klassische Antikörper und Small-molecule- Tyrosinkinaseinhibitoren}

Zielgerichtete Therapeutika revolutionieren die Onkologie aufgrund ihrer hohen antitumorösen Effektivität bei deutlich niedriger unspezifischer Zytotoxizität. Trotzdem können auch zielgerichtete onkologische Therapeutika $\mathrm{zu}$ Nebenwirkungen führen, insbesondere, wenn andere Signalwege, sog. „offtargets“, beeinflusst werden. Dies ist bedeutsam gerade bei schlecht regenerierenden Organen und Zelltypen wie Herz und Blutgefäße. Kardiovaskuläre Nebenwirkungen aktueller zielgerichteter onkologischer Therapeutika, die entsprechend der Fachinformation als häufig oder sehr häufig aufgeführt werden, zeigt - Tab. 3.

\section{Tyrosinkinaseinhibitoren mit multiplen Ansatzpunkten}

Die bei vielen Wirkstoffen beobachtete Blutdruckerhöhung ist mit der On-target-Wirkung auf den VEGFR-Signalweg gut erklärt. Inwieweit zusätzliche Effekte auf die Gefäßstruktur/Gefäßsteifigkeit eine Rolle spielen, die z.B. für Sorafenib und Sunitinib beschrieben wurden, ist unklar. Viele Tyrosinkinaseinhibitoren haben durch unspezifische Bindung an die ATP-Bindungsstelle anderer Kinasen multiple, sogenannte Off-targetEffekte. Einige der inhibierten Signalwege wie RAF1 und KIT regulieren Überleben und Reparaturmechanismen in Herzmuskelzellen, kardiotoxische Wirkungen wurden sowohl in kultivierten Herzmuskelzellen wie auch in Tierexperimenten nachgewiesen [133]. Die Angaben zum Auftreten einer LVDysfunktion unter Sorafenib und Sunitinib schwanken zwischen 1 und $9 \%$ [52, 100, 102]. Der ausgeprägteste Effekt unter den Tyrosinkinaseinhibitoren auf die Verlängerung der QTc ist für Vandetanib beschrieben mit dosisabhängigen Raten von 3-12\% [45]. Ein statistischer Zusammenhang der QTcVerlängerung mit insgesamt sehr selten aufgetretenen, malignen Arrhythmien konnte unter Studienbedingungen nicht beobachtet werden. Allerdings werden in der Routineanwendung in $24-74 \%$ Medikamente mit Interaktionspotenzial über den Cytochrom-P450 3A4-Metabolismus verschrieben, was zu erhöhtem Toxizitätspotenzial führen kann. Für Vandetanib wurde in vitro eine direkte Bindung an kardiomyozytäre hERGKaliumkanäle und eine Inhibition von Kalium- und Natriumströmen gezeigt, was mit einer attenuierten Depolarisation und einer Verzögerung des Aktionspotenzials einherging [75].

\section{BCR-ABL-Inhibitoren}

$\mathrm{Zu}$ den ersten Kinaseinhibitoren überhaupt gehört Imatinib, das selektiv auf die BCR-ABL-Translokation wirkt, welche als Fusionskonstrukt die treibende Kraft bei chronischer myeloischer Leukämie ist. Relevante Häufungen kardiovaskulärer Nebenwirkungen wurden unter Imatinib kaum beschrieben. Die BCRABL-Inhibitoren der folgenden Generationen sind deutlich effizienter, zeigten aber eine Reihe von kardiovaskulären Nebenwirkungen. Unter Dasatinib wurde bei $3 \%$ der Patienten eine pulmonalarterielle Hypertonie beobachtet [51], die bei ca. zwei Drittel der Pa- 
Tab. 3 Onkologische Indikation, kardiovaskuläre Nebenwirkungen und Therapiemonitoring ${ }^{a}$ nach Fachinformation: (sehr häufig >10\%, häufig $1-10 \%)$

\begin{tabular}{|l|l|l|}
\hline Indikation & $\begin{array}{l}\text { Peri-/ } \\
\text { myokardiale } \\
\text { Erkrankungen }\end{array}$ & Arrhythmien \\
\hline
\end{tabular}

\section{Small-molecule-Tyrosinkinaseinhibitoren \\ $B C R-A B L$}

Imatinib (PDGFR, KIT)

$\mathrm{CML}, \mathrm{ALL}$, gastrointestinale Stromatumoren, chronische eosinophile Leukämie

Dasatinib (SRC)
Nilotinib (KIT, PDGFR)
Bosutinib (SRC)
Ponatinib (VEGFR,
PDGFR, SRC)
BRAF

Vemurafenib

Dabrafenib

Encorafenib

CML, ALL

CML

CML

CML, ALL

Melanom, Haarzellleukämie, multiples Myelom

MEK

Trametini

Cobimetinib

Binimetinib

ALKMET

Crizotinib

Alectinib

Brigatinib

Ceritinib

Bruton

Ibrutinib

Acalabrutinib

EGFR

Erlotinib

Gefitinib

Lapatinib (HER2)

Afatinib

Osimertinib

Neratinib (HER2)

Multi-Target

Sorafenib

(RAF-1/B-RAF, VEGFR2, PDGFR)

Sunitinib (VEGFR, PDGFR, KIT)

Pazopanib (VEGFR, PDGFR, KIT)

\section{Melanom, NSCLC}

Melanom

\section{Melanom, NSCLC}

Melanom

Melanom

NCSLC

NSCLC

NSCLC

NSCLC

CLL, Mantelzelllymphom, Morbus Waldenström

Lymphoplasmozytisches Lymphom, Mantelzelllymphom, CLL

NSCLC, Pankreaskarzinom

NSCLC

Mamma-Ca

NSCLC

NSCLC

Mamma-Ca

HCC, RCC, follikuläres SD-Karzinom

$\mathrm{HI}$

GIST, RCC, neuroendokriner Pankreastumor

RCC, Weichteilsarkom
HI, Perikarderguss

Perikarderguss

HI, Perikarderguss

QTc-Verlängerung

QTC-Verlängerung, VHF, AV-Block

QTC-Verlängerung

VHF

-
LV-Dysfunktion
LV-Dysfunktion

QTc-Verlängerung

$-$

SVT

LV-Dysfunktion

LV-Dysfunktion

LV-Dysfunktion

HI

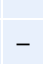

$-$

Perikarditis

Tachykardie, Bradykar-
Bradykardie

$-$

$-$

Bradykardie, QTc-Verlängerung

Bradykardie die, QTc-Verlängerung

Bradykardie, QTc-Verlängerung

VHF, ventrikuläre Tachyarrhythmien
Vaskuläre Er- Monitoring krankungen

$\begin{array}{ll}\text { ATE, HTN } & \begin{array}{l}\text { LKG } \\ \text { sipide, Ba- } \\ \text { sis-EKG }\end{array} \\ \text { HTN } & \text { Basis-EKG } \\ \text { HTN, VTE, ATE } & \text { RR }\end{array}$

Vaskulitis

HTN

HTN, VTE TTE, EKG, Elyte

\begin{tabular}{l|l} 
HTN & TTE, RR \\
\hline HTN & TTE \\
\hline HTN, VTE & RR, TTE
\end{tabular}

EKG, Elyte, RR HF/RR EKG EKG

\begin{tabular}{l|l}
- & EKG \\
\hline- & - \\
\hline & \\
- & - \\
- & - \\
\hline- & TTE, EKG \\
\hline- & - \\
\hline- & - \\
\hline- & - \\
\hline MI, HTN & RR \\
\hline Ischämie, HTN, & RR \\
\hline VTE & RR, EKG, \\
\hline HTN, VTE & Elyte \\
\hline
\end{tabular}




\begin{tabular}{|c|c|c|c|c|c|}
\hline & Indikation & $\begin{array}{l}\text { Peri-/ } \\
\text { myokardiale } \\
\text { Erkrankungen }\end{array}$ & Arrhythmien & $\begin{array}{l}\text { Vaskuläre Er- } \\
\text { krankungen }\end{array}$ & Monitoring \\
\hline $\begin{array}{l}\text { Vandetanib (VEGFR, } \\
\text { EGFR) }\end{array}$ & Medulläres SD-Karzinom & - & QTc-Verlängerung & HTN & $\begin{array}{l}\text { EKG, Elyte, } \\
\text { RR }\end{array}$ \\
\hline $\begin{array}{l}\text { Lenvatinib (VEGF, FGFR, } \\
\text { PDGF, KIT, RET) }\end{array}$ & RCC, HCC, follikuläres SD-Karzinom & $\mathrm{HI}$ & QTc-Verlängerung & HTN, MI, VTE & $\begin{array}{l}\text { RR, EKG, } \\
\text { Elyte }\end{array}$ \\
\hline Regorafenib (VEGFR) & Kolorektal, GIST, HCC & - & - & HTN & $\mathrm{RR}$ \\
\hline Axitinib (VEGFR) & $\mathrm{RCC}$ & $\mathrm{HI}$ & - & HTN, VTE, MI & $\mathrm{RR}$ \\
\hline $\begin{array}{l}\text { Nintedanib (VEGFR, } \\
\text { FGFR, PDGFR) }\end{array}$ & NSCLC & - & - & - & - \\
\hline $\begin{array}{l}\text { Cabozantinib (VEGFR, } \\
\text { MET, RET) }\end{array}$ & RCC, HCC, medulläres SD-Karzinom & - & - & HTN, VTE, ATE & $\begin{array}{l}\text { RR, EKG, } \\
\text { Elyte }\end{array}$ \\
\hline \multicolumn{6}{|l|}{ Antikörper } \\
\hline \multicolumn{6}{|l|}{ HER2 } \\
\hline Pertuzumab & Mamma-Ca & LV-Dysfunktion & - & - & $\begin{array}{l}\text { TTE, Tropo- } \\
\text { nin }\end{array}$ \\
\hline Trastuzumab & Mamma-Ca, Magen-Ca & LV-Dysfunktion/HI & - & - & $\begin{array}{l}\text { EKG, TTE, } \\
\text { Troponin }\end{array}$ \\
\hline $\begin{array}{l}\text { Ado-Trastuzumab } \\
\text { Emtansin }\end{array}$ & Mamma-Ca & LV-Dysfunktion & - & - & $\begin{array}{l}\text { TTE, Tropo- } \\
\text { nin }\end{array}$ \\
\hline \multicolumn{6}{|l|}{ VEGF } \\
\hline Bevacizumab & $\begin{array}{l}\text { Kolon-Ca, Mamma-Ca, NSCLC, RCC, } \\
\text { Ovar-Ca, Zervix-Ca }\end{array}$ & $\mathrm{HI}$ & SVT & HTN, VTE, ATE & $\mathrm{RR}$ \\
\hline Ramucirumab & Magen, Kolorektal, NSCLC & - & - & HTN & $\mathrm{RR}$ \\
\hline Aflibercept & Kolorektal & - & - & HTN, VTE, ATE & $\mathrm{RR}, \mathrm{TTE}$ \\
\hline \multicolumn{6}{|l|}{$E G F R$} \\
\hline Panitumumab & Kolorektal & - & Tachykardie & VTE, HTN & - \\
\hline Cetuximab & $\begin{array}{l}\text { Kolorektal, Plattenepithel-Ca Larynx/ } \\
\text { Pharynx }\end{array}$ & - & - & - & - \\
\hline Necitumumab & Plattenepithel-Ca, NCSLC & - & - & VTE, ATE & - \\
\hline \multicolumn{6}{|c|}{ 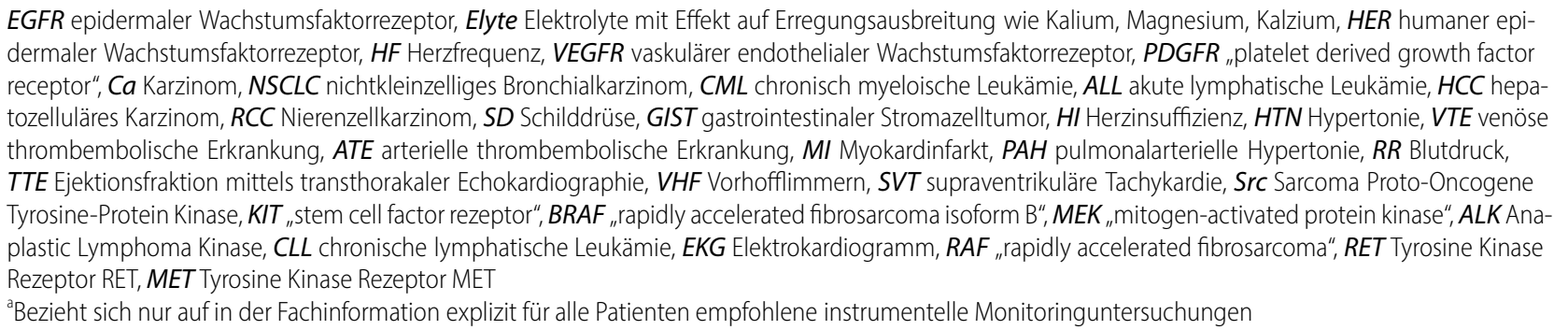 } \\
\hline
\end{tabular}

tienten nach Absetzen reversibel ist, aber auch dauerhafte und klinisch fatale Verläufe verursachen kann [124]. Ursächlich scheinen Off-target-Effekte von Dasatinib an SRC-Kinasen, die die Proliferation von glatten Gefäßmuskelzellen und Vasokonstriktion regulieren. Zusätzlich werden endothelschädigende Effekte über ROS diskutiert [51]. Therapeutika zur Behandlung der pulmonalarteriellen Hypertonie scheinen auch bei Dasatinib-induzierten Fällen zu wirken [145]. Nilotinib zeigt dosis- und zeitabhängig arterielle Gefäßverschlüsse in über $10 \%$ bei 6-jähriger Behandlung [71]. Die molekularen Mechanismen sind weitestgehend unklar. Aufgrund ungünstiger metabolischer Effekte mit Hyperglykämie und Hyperlipidämie und sekundär akzelerierter Atherosklerose werden eine Kontrolle der Fettstoffwechselparameter vor Therapie, und nach 3, 6 und alle 12 Monate sowie eine Kontrolle der Blutglukose empfohlen. Bei anderen Patienten treten die Veränderungen rasch ein und könn- ten durch experimentell nachweisbare direkte apoptotische und proatherogene Effekte auf vaskuläre Endothelzellen erklärbar sein. Klassische kardiovaskuläre Risikofaktoren erhöhen das Risiko vaskulärer Nebenwirkungen, und etablierte kardiologische Risikoscores wie SCORE können auch bei Patienten mit Nilotinib das Risiko für vaskuläre Ereignisse abschätzen [114]. Ponatinib als Inhibitor der 3. Generation und Multikinaseinhibitor hat dosisabhängig neben VEGFRInhibition-vermittelter Hypertension 
v. a. ein erhöhtes Risiko für vaskuläre thrombembolische Komplikationen mit schweren arteriellen thrombotischen Ereignissen bei über $20 \%$ der Patienten [27]. Ponatinib erzeugt experimentell Mikroangiopathien [72] und einen prothrombogenen Zustand über die Aktivierung inflammatorischer Mediatoren wie TNF- $\alpha$, Interferon- $\gamma$, Interleukin- 6 und P-Selectin [54]. Wichtig ist, dass gerade für schwer therapierbare Formen der akuten lymphatischen Leukämie auch Kombinationstherapien empfohlen werden. Zum Beispiel erhöht sich die Effizienz von Dasatinib, wenn es mit M199 (Venetoclax), einem selektiven Inhibitor des B-cell-lymphoma 2(BCL2)-Proteins [79] oder noch zusätzlich mit Dexamethason [122] kombiniert wird. Analysen zur Wirkung solcher Kombinationstherapien auf das kardiovaskuläre System sind dringend notwendig.

\section{BRAF- und MEK-Inhibitoren}

Die Hemmung des Ras-Raf-MEK1ERK1/2-Signalwegs kann zu einer Einschränkung der LV-Funktion, zu Blutdruckanstieg und zu einer Verlängerung der QTc führen. Vor allem unter einer Kombinationstherapie mit BRAF- und MEK-Inhibitor sind der Abfall der LVEF und das Auftreten von Hypertonie häufiger mit $8,1 \%$ vs. $2 \%$ und $19,5 \%$ vs. $14 \%$ verglichen mit BRAF-Monotherapie [93]. Für Cobimetinib wird ein Monitoring der LVEF nach 1 Monat und dann 3-monatig empfohlen und bei Abfall der LVEF (<40\% oder $40-49 \%$ und $\mathrm{um} \geq 10 \%$ ) eine Therapiepause bzw. ein Absetzen (anhaltend $<40 \%$ ) angeraten. Zugrunde liegende Mechanismen sind weitestgehend unklar. Ein Effekt des BRAF-MEK-Weges auf kardiovaskulär exprimierte MAP-Kinasen wird vermutet, die unter physiologischen Bedingungen kardioprotektive Effekte vermitteln und die Stickstoffmonoxidproduktion aktivieren.

\section{ALK-Inhibitoren}

Die elektrophysiologischen Effekte der ALK-Inhibitoren gehen auf direkte Effekte auf multiple kardiomyozytäre Ionenkanäle zurück [38, 150]. Klinisch werden v. a. Sinusbradykardien beobachtet, die meist milde oder sogar asymptomatisch verlaufen [107]. Die maximale Reduktion der Herzfrequenz unter Alectinib- und Crizotinib-Therapie lag durchschnittlich bei $13 / \mathrm{min}$ und $25 / \mathrm{min}$ und trat mehrere Wochen nach Therapiebeginn auf. Der stärkste Prädiktor für das Auftreten einer Sinusbradykardie unter Therapie war eine niedrigere Ausgangsherzfrequenz (<70/min). Symptomatische Bradykardien bessern sich nach Dosisreduktion der ALK-Inhibitoren.

\section{Bruton-Kinase-Inhibitor}

Unter Ibrutinib wurde eine Inzidenz für Vorhofflimmern von 3,3\% beschrieben. Realworld-Daten zeigen im ersten Jahr der Behandlung sogar eine kumulative Inzidenz von 7,5\% [147], was aufgrund des paroxysmalen Charakters von Vorhofflimmern, dem oft asymptomatischen Verlauf und der retrospektiven Analyse vermutlich unterschätzt wird. Tierexperimentell führt Ibrutinib zu strukturellem Remodelling und Störung der Kalziumhomöostase am Vorhofmyokard, was zur Ausbildung von Vorhofflimmern beitragen kann [62]. Sowohl die Bruton-Kinase als auch die „off-target“ beeinflusste Tec-Proteintyrosinkinase regulieren den Phosphoinositid 3-Kinase(PI3K)-Akt-Signalweg, der sowohl für Kardioprotektion wie auch kardiale Hypertrophie eine wichtige Rolle spielt und z. B. bei Patienten mit Vorhofflimmern herunterreguliert ist. Eine besondere Herausforderung stellt die Behandlung von Patienten mit Vorhofflimmern unter Ibrutinib dar, da es wegen seiner Metabolisierung über Zytochrom P4503A4 bei einer Komedikation mit Verapamil oder Amiodaron zu massiven Spiegelanstiegen von Ibrutinib kommen kann [43]. Umgekehrt steigen die Spiegel der direkten oralen Antikoagulanzien, v. a. des Thrombininhibitors Dabigatran, unter Ibrutinib an und erhöhen so das Blutungsrisiko. Unabhängig davon beeinflusst Ibrutinib verschiedene Thrombozytensignalwege und erhöht so grundsätzlich das Blutungsrisiko. Die Entscheidung zur Antikoagulation sollte deshalb individuell unter Berücksich- tigung des Schlaganfall-, v. a. aber des Blutungsrisikos erfolgen.

\section{HER2-Inhibitoren/EGF2-abhängige Therapeutika}

Der monoklonale Antikörper Trastuzumab ist eines der ältesten zielgerichteten Therapeutika in der Onkologie und wird v. a. bei der Behandlung des Mammakarzinoms eingesetzt. Dementsprechend gibt es hierfür viel Erfahrung und im Gegensatz zu den meisten anderen Wirkstoffen konkrete Empfehlungen zum onkokardiologischen Management. Der Signalweg des epidermalen Wachstumsfaktor(EGF)-Rezeptors 2 spielt in Kardiomyozyten eine wichtige Rolle bei der Kompensation von Stress- oder Schädigungsreizen, indem kardioprotektive subzelluläre Mechanismen aus dem Bereich der Energiehomöostase, Kalziumregulation, Inotropie und Ultrastruktur aktiviert werden. Zusätzlich verursacht eine Inhibition des HER2abhängigen Signalweges tierexperimentell kardiomyozytäre Apoptose [14]. Die bedeutendste klinische kardiovaskuläre Komplikation von EGF-abhängigen Therapeutika wie den Antikörpern Trastuzumab/Pertuzumab und dem dualen Kinaseinhibitor Lapatinib sind die LVDysfunktion und klinisch manifeste Herzinsuffizienz. Trastuzumab ist die Leitsubstanz der „Typ-2“-Kardiotoxizität, die in Abgrenzung zur Anthrazyklinbedingten Typ-1-Kardiotoxizität als dosisunabhängig, meist reversibel und ohne Langzeiteffekte galt. Diese historische Einteilung gilt heute größtenteils als überholt, da sich die Toxizitätscharakteristika der beiden Substanzklassen deutlich überschneiden.

In den frühen Trastuzumab-Studien wurden Herzinsuffizienz und LV-Dysfunktion bei bis zu $30 \%$ der Patienten beobachtet [126]. Aufgrund der verbesserten Patientenauswahl, optimierter Therapieregime und des kardialen Monitorings unter Therapie werden heute Ereignisraten von unter $10 \%$ für Trastuzumab beobachtet [48] und niedrigere Raten bei Pertuzumab, Emtansin und Lapatinib [61]. Wichtigste Risikofaktoren für eine Trastuzumab-assoziierte Kardiotoxizität sind eine vorausgegangene oder 
synchrone Anthrazyklin-Therapie oder eine vorbestehende eingeschränkte LVEF [104]. Die Erholungsrate der Trastuzumab-assoziierten LV-Dysfunktion liegt bei ca. $80 \%$ [148].

Für Trastuzumab und ähnlich für die anderen ERB2-Inhibitoren werden eine klinische Evaluation, EKG und eine Bestimmung der LVEF vor und alle 3 Monate unter Therapie sowie alle 6 Monate bis 2 Jahre nach Therapieende empfohlen. Bei einem Abfall der LVEF von $10 \%$ oder mehr auf unterhalb von $50 \%$ werden eine Pausierung der Therapie und Reevaluation nach 3 Wochen empfohlen. Dies deckt sich weitestgehend mit dem aktuellen Positionspapier der ESC, in dem allerdings bei einem Abfall der LVEF auf Werte zwischen 45 und $49 \%$ noch eine Therapiefortführung unter einer Gabe von ACE-Hemmern als möglich erachtet wird. Letzteres wird unterstützt durch eine aktuelle Beobachtungsstudie, in der eine Therapie mit Trastuzumab oder Pertuzumab bei Patientinnen mit Mammakarzinom und leichter, asymptomatischer linksventrikulärer Dysfunktion (LVEF 40-49\%) unter einer Vorbehandlung mit ACE-Hemmer und $\beta$-Blocker und kardiologischer Kontrolle in $90 \%$ der Patienten ohne kardiovaskuläre Ereignisse durchgeführt werden konnte [83]. Die klinische und medizinökonomische Effektivität eines Monitorings überhaupt und bestimmter Monitoringintervalle wurde bisher nicht prospektiv evaluiert.

Eine primärpräventive, kardioprotektive Therapie wird aktuell kontrovers diskutiert [10] und im europäischen Positionspapier für nicht näher definierte Hochrisikopatienten empfohlen. Die größte randomisierte Studie an $468 \mathrm{~Pa}$ tienten mit Trastuzumab-Therapie fand einen signifikanten Vorteil einer präventiven ACE-Hemmer- und $\beta$-Blocker-Behandlung im Vergleich zu Plazebo nur bei Patienten, die zusätzlich mit Anthrazyklinen behandelt wurden [50].

Tritt eine Herzinsuffizienz mit LVDysfunktion unter Trastuzumab auf, wird eine Behandlung entsprechend den kardiologischen Leitlinien für Herzinsuffizienz empfohlen.

\section{VEGFR-Inhibitoren}

Eine Inhibition des Signaltransduktionswegs des vaskulären endothelialen Wachstumsfaktorrezeptors (VEGFR) wirkt über Angiogenesehemmung antitumorös und kann antikörpervermittelt auf Basis des Wachstumsfaktors oder des Rezeptors erfolgen oder über Inhibition nachgeschalteter Tyrosinkinasen. Die bedeutendste kardiovaskuläre $\mathrm{Ne}$ benwirkung aller VEGFR-Inhibitoren ist die Blutdrucksteigerung. Bei bis $\mathrm{zu}$ $80 \%$ der behandelten Patienten wird eine Hypertonie beobachtet, die dosisabhängig und meist nach Absetzen der Therapie reversibel ist. Wenngleich der Blutdruckanstieg mit der antitumorösen Therapieeffektivität korreliert, beeinträchtigt umgekehrt eine medikamentöse Senkung des Blutdrucks nicht die Therapiewirkung auf den Tumor. Die Blutdrucksteigerung wird über eine Hemmung vasodilatierender Faktoren wie Stickstoffmonoxid und Prostaglandinen und eine Steigerung von vasokonstriktorischen Faktoren wie Endothelin sowie über eine Reduktion der Kapillargefäßdichte vermittelt [37]. Aufgrund der raschen Blutdrucksteigerung nach Therapiebeginn mit VEGFR-Inhibitoren und fehlender Adaptationsmechanismen ist sekundär das Risiko für vaskuläre Ereignisse wie Schlaganfall oder Myokardinfarkt erhöht. LV-Funktionsstörungen und Herzinsuffizienz traten in randomisierten Studien mit VEGFRInhibitoren in 2,4\% auf [46], wobei die Rate in der klinischen Routine höher zu sein scheint [102]. Neben direkten kardiotoxischen Effekten der VEGF-Inhibitoren begünstigt die Hypertension die Ausbildung der LV-Dysfunktion, die zumindest zum Teil reversibel zu sein scheint. Der QTc verlängernde Effekt variiert sehr stark zwischen den VEGFRInhibitoren und scheint auf direkten Effekten auf myokardiale Kaliumkanäle zu beruhen [65].

Vor Beginn einer VEGFR-InhibitorBehandlung sollte im Rahmen der kardiovaskulären Basiserhebung besonders auf eine optimale Blutdruckeinstellung geachtet werden. Als mögliches Monitoringregime wurden von dem National Cancer Institute Blutdruckkontrollen während des ersten Zyklus wöchentlich und im Anschluss alle 2 bis 3 Wochen vorgeschlagen [129]. Die Behandlung der Hypertonie erfolgt grundsätzlich gemäß den entsprechenden kardiologischen Leitlinienempfehlungen. Aufgrund von Interaktionen der meisten VEGFR-Inhibitoren mit Zytochrom P450 3A4 sollten Dihydropyridin-Kalziumantagonisten vermieden werden. Diuretika haben das Risiko eines Elektrolytverlustes, was eine QTc-Verlängerung begünstigen kann, und sind deshalb nur mit Vorsicht einzusetzen. Mittel der ersten Wahl sind ACEHemmer/AT1-Inhibitoren, $\beta$-Blocker und Nicht-Dihydropyridin-Kalziumantagonisten. Bei Behandlungsregimen mit therapiefreien Intervallen muss auf eine Reboundhypotension geachtet werden und die antihypertensive Therapie ggf. angepasst werden. Evidenz bezüglich eines routinemäßigen Screenings auf LV-Dysfunktion gibt es bislang nicht. Eine konkrete Monitoringempfehlung der QTc besteht nach Fachinformation bislang nur für Vandetanib, wo ein Ausgangs-EKG und EKG- und Elektrolytkontrollen nach 1, 3, 6 und 12 Wochen sowie dann alle 3 Monate empfohlen werden. Eine Ausgangs QTc von mehr als $480 \mathrm{~ms}$ ist eine Kontraindikation für Vandetanib.

\section{Neue hämatologische Therapien}

$\mathrm{Zu}$ den neueren hämatologischen Therapien zählen immunmodulatorische $\mathrm{Me}$ dikamente, Antikörpertherapien (CD38Antikörper, CD20-Antikörper, CD79bAntikörper, CD30-Antikörper, Slam-F7Antikörper, CD22-Antikörper, CD3/19Antikörper) sowie Hedgehog-Signalweginhibitoren und PI3K-Inhibitoren (- Tab.4). Der folgende Abschnitt fokussiert auf hämatologische Medikamente aus den Gruppen der Proteasominhibitoren, der Histon-Deacetylase(HDAC)Inhibitoren sowie der immunmodulatorischen Medikamente, da diesbezüglich spezifische kardiale Nebenwirkungen berichtet wurden und ein Hinweis zur Diagnostik und Therapie gegeben werden kann. Bei den anderen neueren hämatologischen Therapien müssen umfangreichere Analysen abgewartet 
Tab. 4 Überblick über neue hämatologische Medikamente, Indikation sowie aus klinischer/ kardiologischer Perspektive die häufigste potenzielle kardiale Nebenwirkung

\begin{tabular}{|c|c|c|c|}
\hline $\begin{array}{l}\text { Wirkstoff- } \\
\text { gruppe }\end{array}$ & Wirkstoff & Aktuelle Zulassung $^{\mathrm{a}}$ & $\begin{array}{l}\text { Potenzielle kardiale Nebenwir- } \\
\text { kungen }\end{array}$ \\
\hline \multirow{2}{*}{$\begin{array}{l}\text { Proteasom- } \\
\text { inhibitoren }\end{array}$} & Bortezomib & Multiples Myelom & Herzinsuffizienz \\
\hline & Carfilzomib & Multiples Myelom & Herzinsuffizienz \\
\hline \multirow[t]{3}{*}{$\begin{array}{l}\text { HDAC-Inhibi- } \\
\text { toren }\end{array}$} & Vorinostat & $\begin{array}{l}\text { Kutanes T-Zell-Lym- } \\
\text { phom, multiples Mye- } \\
\text { lom }\end{array}$ & QTc-Verlängerung \\
\hline & Panobinostat & Multiples Myelom & QTc-Verlängerung \\
\hline & Romidepsin & - & QTc-Verlängerung \\
\hline \multirow[t]{2}{*}{$\begin{array}{l}\text { Immunmodula- } \\
\text { torische } \\
\text { Medikamente }\end{array}$} & Lenalidomid & Multiples Myelom & $\begin{array}{l}\text { Arterielle/venöse Thrombosen, } \\
\text { arterielle Hypertonie, Herzinsuffi- } \\
\text { zienz }\end{array}$ \\
\hline & Pomalidomid & Multiples Myelom & Arterielle/venöse Thrombosen \\
\hline \multicolumn{4}{|c|}{$\begin{array}{l}\text { HDAC Histon-Deacetylasen } \\
{ }^{\circ} \text { Gemäß EMA (Europäische Arzneimittelagentur), Zulassung teils erst als Möglichkeit in der Zweit- } \\
\text { oder Drittlinientherapie }\end{array}$} \\
\hline
\end{tabular}

werden, bevor eine Beurteilung potenziell kardiotoxischer Effekte erfolgen kann.

\section{Proteasominhibitoren}

Neben reversiblen Inhibitoren des Proteasoms (beispielsweise das Bortezomib) wurden irreversible Inhibitoren mit deutlich verlängerter und verbesserter Wirkung am Proteasom entwickelt (beispielsweise Carfilzomib) [55]. Carfilzomib hat sich als eine Option in der Zweitlinientherapie des multiplen Myeloms etabliert [36, 131]. In den $\mathrm{Zu}-$ lassungs- und Toxizitätsstudien zeigen die irreversiblen Inhibitoren schon nach kurzer Zeit eine fast komplette Inhibition der Proteasomaktivität in Kardiomyozyten. Zudem wurde bei allen getesteten Dosierungen im präklinischen Modell eine Inflammation im Herzen histologisch nachgewiesen. Eine Reduktion der LVEF wurde bereits im Rahmen präklinischer Studien berichtet [103].

\section{Klinische Datenlage}

Irreversible Proteasominhibition ist mit einer erhöhten Rate von kardiovaskulären Ereignissen, insbesondere Herzinsuffizienz verbunden. In der bisher größten klinischen Studie traten bei $22 \%$ der Patienten kardiale Nebenwirkungen auf (Arrhythmien, meist Vorhofflimmern [13,3\%]; Herzinsuffizienz [7,2\%]; behandlungsassoziierte Kardiomyopathie [2\%]; myokardiale Ischämien [3\%])
NT-proBNP oder Echokardiographie erscheint aber nicht möglich [31, 118].

\section{Empfehlung zur Kardiotoxizität}

Patienten mit eingeschränkter LVEF oder Vorhofflimmern sollten engmaschig betreut werden. Kardiale Biomarker (insbesondere Troponin) haben wahrscheinlich einen prädiktiven Wert, erhöhte Werte sollten ebenfalls eine engmaschigere Betreuung zur Folge haben [91]. Eine Beurteilung erfolgt gemäß den Empfehlungen zur Diagnostik und Therapie der Herzinsuffizienz der ESC [111].

Insbesondere während der ersten 3 Monate scheinen sich kardiovaskuläre Nebenwirkungen zu manifestieren, sodass Patienten mit erhöhtem Risiko (kardiale Vorerkrankung, umfangreiche stattgehabte onkologische Therapien) innerhalb der ersten 3 Monate nach Initialisierung einer Therapie kardiologisch betreut werden sollten.

\section{Vorgehensweise bei vermuteter Kardiotoxizität}

Bei Verdacht auf eine Carfilzomib-assoziierte Reduktion der LVEF sollte die Therapie zunächst unterbrochen werden und eine Herzinsuffizienztherapie gemäß den Leitlinien der ESC initialisiert werden [111].

Im weiteren Verlauf kann unter einer strengen Kontrolle der kardialen Biomarker und der LVEF eine erneute Therapie mittels Carfilzomib, ggf. in reduzierter Dosierung versucht werden [111].

\section{Histondeazetylasen(HDAC)- Inhibitoren}

HDAC-Inhibitoren wirken durch Bindung und Inhibition der DeazetylaseDomäne von Histon-modifizierenden Proteinen. Die aktuell zugelassenen Medikamente gehören zu den Pan-Inhibitoren und sind a. e. unspezifische Inhibitoren mit geringer Affinität zu bestimmten HDACs. Die pharmakologische Inhibition der HDACs führt aber nicht nur zu einer Modifikation des Epigenoms, vielmehr gibt es zahlreiche nichtnukleäre Zielproteine der HDACs, sodass die Wirkung nicht ausschließlich auf eine transkriptionelle Modifikation beschränkt ist. Unter den zahlreichen 
HDAC-Inhibitoren sind bisher nur wenige zur klinischen Anwendung in onkologischen Therapien zugelassen. Vorinostat ist zur Behandlung des refraktären und fortgeschrittenen kutanen T-ZellLymphoms zugelassen, Panobinostat zur Behandlung des refraktären und/oder rezidivierenden multiplen Myeloms nach mindestens 2 vorausgegangenen Therapien.

In den initialen klinischen Studien mit HDAC-Inhibitoren gab es Berichte von supraventrikulären Arrhythmien [123, 134]. Veränderungen der LVEF oder erhöhte kardiale Biomarker zeigten sich nicht.

Aufgrund der vorliegenden Daten stehen bei HDAC-Inhibitoren die potenziellen proarrhythmogenen Effekte im Vordergrund. $\mathrm{Zu}$ den frühen Zeichen von EKG-Veränderungen gehören $\mathrm{T}$-Negativierung sowie QTc-Verlängerungen [134]. Bei Letzteren sollte unter der Therapie eine Normalisierung bis zur Weiterführung abgewartet werden sowie eine Kontrolle der Elektrolytwerte erfolgen. Eine Komedikation mit potenziell QTc verlängernden Medikamenten sollte vermieden werden. Präklinische Daten weisen bezüglich kardialer Fibrose und bezüglich der linksventrikulären Pumpfunktion auf potenziell kardioprotektive Mechanismen hin [76].

\section{Immunmodulatorische \\ Medikamente}

Die Klasse der immunmodulatorischen Medikamente wirken über mehrere molekulare Mechanismen (Stimulation von T-Zellen, Hemmung der Proliferation hämatopoetische Zellen, Antiangiogenese) an Tumorzellen. Eine Hochregulation von TNF- $\alpha$ wird neben einer veränderten Ausschüttung endothelialer Botenstoffe mit einer erhöhten Neigung zu Thrombosen in Verbindung gebracht. Die beiden am häufigsten eingesetzten Wirkstoffe sind Lenalidomid und Pomalidomid, sie leiten sich strukturell von Thalidomid ab.

In den größeren Studien zeigen sich v. a. eine Häufung thrombembolischer Ereignisse sowie Überleitungsstörungen $[42,66]$. Die genaue Häufigkeit der Ereignisse unter Therapie mit immunmodu- latorischen Medikamente ist unklar und schwankt je nach Berichten der klinischen Studien stark [74]. Das Auftreten einer Thrombose oder eines thrombembolischen Ereignisses muss als mögliche Nebenwirkung bei bis zu 23 \% der Patienten erwartet werden, insbesondere unter Kotherapie mit Erythropoietin [35, 66]. Von einer Herzinsuffizienz wird bei bis zu $4 \%$, von einer arteriellen Hypertonie bei bis zu $6,9 \%$ der Patienten berichtet [74]. Im Vergleich zur Behandlung mit Bortezomib zeigt sich bei Patienten mit multiplem Myelom und Lenalidomid-Therapie allerdings keine weitere Steigerung kardiovaskulärer Ereignisse [115]. Neben den thrombembolischen Ereignissen werden gehäuft Sinusbradykardien sowie Vorhofflimmern berichtet [40]. Sinusbradykardien treten unabhängig von sonstigen EKG-Veränderungen bzw. Verlängerungen der Überleitungszeiten auf. Gegebenenfalls sollte eine zusätzliche bradykardisierende Medikation reduziertbzw. nur nach erneuter Kontrolle der Herzfrequenz initialisiert werden.

Aufgrund der deutlichen Häufung thrombembolischer Komplikationen einer immunmodulatorischen Therapie (Thalidomid und Lenalidomid) wird die prophylaktische Gabe von Thrombozytenaggregationshemmern (Aspirin $100 \mathrm{mg}$ ) bei Patienten ohne Risikofaktoren für eine Thrombose und eine Gabe von Heparin bzw. Marcumar bei zusätzlichem Thromboserisiko, vom Europäischen Myelom-Netzwerk sowie von der Europäischen Gesellschaft für medizinische Onkologie (ESMO) empfohlen [95, 139]. Die Entscheidung über die Maßnahme sollte individuell angepasst sein (mögliche Kontraindikationen für eine Antikoagulation bzw. Thrombozytenaggregationshemmung). Die Patienten sind zudem anzuweisen, auf klinische Symptome einer möglichen Thrombose $\mathrm{zu}$ achten und thromboseförderndes Verhalten einzuschränken (z.B. Rauchen). Unter der zusätzlichen Gabe von Erythropoietin ist das Thromboserisiko weiter erhöht.

\section{CAR-T-Zelltherapien}

Auch bei den neu etablierten CART-Zelltherapien scheint ein fokussier- tes kardiales Monitoring indiziert $\mathrm{zu}$ sein. Obwohl bislang keine Daten aus größeren multizentrischen Erhebungen zur Verfügung stehen und die Inzidenz kardialer Nebenwirkungen im Rahmen dieses Therapieansatzes noch nicht näher definiert ist, berichten einzelne onkokardiologische Arbeitsgruppen über kardiale Nebenwirkungen als möglichen On-target/off-tumor-Effekt der CAR-TZelltherapie.

In Analogie zu den bekannten Nebenwirkungen wie „cytokine release syndrome“ (CRS) oder „immune effector cell-associated neurotoxicity syndrome" (ICANS) sollte die Entwicklung eines bislang nicht beschriebenen „immune effector cell-associated cardiotoxicity syndrome" (OCACS) in das potenzielle Nebenwirkungsspektrum einbezogen werden.

\section{Krebserkrankungen im Kindes- und Jugendalter und ihre Spätfolgen}

\section{Grundlagen}

Onkologische Erkrankungen treten mit einer Inzidenz von 16,8 pro 100.000 bei Kindern und Jugendlichen unter 15 Jahren auf. Das bedeutet in Deutschland 1800 bis 2000 Neuerkrankungen pro Jahr in dieser Altersgruppe. Die akuten Leukämien sind mit $34 \%$ die häufigste Erkrankung, gefolgt von Hirntumoren mit $22 \%$ und Lymphomen mit $12 \%$.

Durch die Erfolge in der Optimierung der Therapie überleben $83 \%$ der pädiatrischen Patienten mindestens 10 Jahre und erreichen das Erwachsenenalter. Damit erhöht sich die Zahl der Langzeitüberlebenden um ca. 1500 Patienten jährlich. Derzeit leben in Deutschland mehr als 30.000 erwachsene Menschen nach durchgemachter onkologischer Erkrankung im Kindesalter.

Bei $60-70 \%$ der ehemaligen jugendlichen Patienten werden Spätfolgen berichtet [105]. Dies sind Kardiomyopathien, aber auch Hörverluste, eingeschränkte Nierenfunktion, endokrine Störungen und Infertilität, Zweitmalignome, neuropsychologische Störungen. Außer der Sorge um eine erneute Tumorerkrankung spielt die Kardiotoxizität für die Progno- 
se eine wichtige Rolle. Die wahrscheinlichste, weil häufigste kardiale Noxe ist dabei die Anthrazyklin-Gabe. Bestrahlung mit dem Thorax im Strahlenfeld ist ebenso von erheblicher Bedeutung. Etwa $60 \%$ der Patienten mit einer onkologischen Erkrankung erhalten Anthrazykline und/oder Bestrahlung. Auch neuere Medikamente wie Tyrosinkinaseinhibitoren kommen in der Pädiatrie in den Fokus der Therapie und werden damit Kandidaten für Nebenwirkungen.

Die Besonderheiten der pädiatrischen Population sind für die Nachsorge im Erwachsenenalter von Bedeutung. Im ESCStatement [149] wird diese Gruppe an 2 Stellen erwähnt: Zum einen erfolgt die Einordnung in die Gruppe mit erhöhtem Risiko für kardiovaskuläre Probleme, zum anderen wird die Notwendigkeit einer lebenslangen Nachsorge konstatiert, da das erhöhte kardiovaskuläre Risiko mindestens 45 Jahre nach Behandlung der onkologischen Erkrankung besteht [94].

\section{Kardiovaskuläre Belastung nach Therapie im Kindes- und Jugendalter}

Folgeprobleme bei onkologischer Erkrankung im Kindesalter sind krankheits- und therapiebedingt zu erwarten. Hauptrisiken für das Herz stellen die Therapie mit Anthrazyklinen und/oder thorakale Bestrahlung dar. Anthrazykline werden unter anderem bei akuten Leukämien, Lymphomen und Knochentumoren eingesetzt [81].

Als Risikofaktoren für Kardiotoxizität durch Anthrazykline im Kindesalter werden genannt:

- hohe kumulative Anthrazyklin-

Dosis,

- hohe Einzeldosis,

- Bolusgabe

- kardiale Vorerkrankungen,

- zusätzliche Bestrahlung,

- junges Alter.

Die Herzinsuffizienz als Spätfolge ist dabei in der Regel mit einer schlechten Prognose verbunden und für die Hälfte der frühzeitigen Todesfälle von Patienten mit pädiatrischen Tumorerkrankungen verantwortlich [19]. Sicher gibt es auch individuelle, genetisch determinierte Toleranz für chemotherapeutisch eingesetzte Medikamente. Varianten im Gen CUGBP Elav-like family member 4 (CELF4) prädisponieren beispielsweise für einen kardialen Schaden nach Anthrazyklin-haltiger Chemotherapie. Versuche an humanen Kardiomyozyten, welche aus induzierten pluripotenten Stammzellen (iPSC-CM) verschiedener Patienten gewonnen wurden, zeigten außerdem, dass individuelle Unterschiede in Bezug auf die Chemotherapeutikatoleranz in iPSC-CM experimentell reproduziert werden konnten [15]. Zur Beurteilung des persönlichen Risikos einzelner Patienten für kardiotoxische Spätfolgen einer onkologischen Behandlung müssen auch individuelle Unterschiede in der Lebensweise, respektive individuelle Lebensereignisse berücksichtigt werden [23].

Es gibt Hinweise darauf, dass eine Schwangerschaft nach einer malignen Erkrankung in der Kindheit und stattgehabter Chemotherapie tatsächlich ein Risiko für die peripartale Kardiomyopathie darstellen könnte [109, 116].

Unterschiede gibt es in der Häufigkeit und Art der kardiologischen Untersuchung und Nachsorge. Van der Pal et al. [143] untersuchten 525 Personen aus einer Gruppe von 601 Langzeitüberlebenden nach einem festen Protokoll. Die echokardiographische Untersuchung bestand im Wesentlichen aus einem Standard-M-Mode. Sie fanden bei $27 \%$ eine subklinische Dysfunktion, definiert als eine LVEF $<30 \%$. Risikofaktoren waren die Anthrazyklin-Dosis, zusätzliche Bestrahlung und das junge Alter.

Im Rahmen der Standardnachsorge sind andere Verfahren wie 3-D-EF, „strain“ sowie die Messung der diastolischen Funktion noch nicht fest etabliert. Gerade diese Methoden scheinen einen frühen Zeitpunkt der kardialen Dysfunktion $\mathrm{zu}$ detektieren [8].

\section{Transition und Aspekte der lebenslangen Nachsorge}

Da kardiale Probleme nach onkologischer Therapie in der Kindheit sich Jahrzehnte später zeigen können, sind eine gute Dokumentation der stattgehabten
Therapie und eine langfristig angelegte Nachsorge zu fordern. Wichtig ist es dabei ein Transitionskonzept und evidenzbasierte Screeningprotokolle $\mathrm{zu}$ entwickeln [142]. Die kardiologische Nachsorge sollte 12 Monate nach Therapieende beginnen und lebenslang mindestens alle 5 Jahre erfolgen [4]. Besonders betreut werden müssen Schwangere. Überlebende der onkologischen Therapie sollten vor und zu Beginn einer Schwangerschaft kardiologisch abgeklärt werden [109].

Ziel der Initiativen in der Kinderheilkunde wird sein, die Patienten mit den nötigen Informationen in die Transition zu schicken und Vorschläge zur Nachsorge zu machen. Vorher ist es erforderlich, diese Patienten nach einem Standardprotokoll zu untersuchen.

Präventionsstrategien sind zu entwickeln, dies muss schon im Rahmen der initialen Therapie beginnen.

Zusammenfassend ist mit einer wachsenden Zahl von Personen zu rechnen, die mit jahrelanger Latenz nach durchgemachter Tumorerkrankung im Kindesalter kardiovaskulär krank werden. Sie müssen mit Informationen ausgestattet sein, die Krankheit, Art und Ausmaß der Therapie beinhalten.

\section{Survivorship-Programme}

Neben der kardiologischen Vorstellung vor Beginn einer potenziell kardiotoxischen onkologischen Behandlung und der regelmäßigen Untersuchung während der Krebstherapie ist die langfristige kardioonkologische Betreuung von Langzeitüberlebenden die dritte Säule der notwendigen Maßnahmen zur Verhinderung oder Reduktion von kardiovaskulären Folgen der Krebstherapie. Mit zunehmenden Erfolgen der Krebstherapie wächst auch die Gruppe der Langzeitüberlebenden, die im Kindesalter oder als Erwachsene kardiotoxischen Therapieverfahren wie hohen AnthrazyklinDosen oder aggressiven Bestrahlungsprotokollen ausgesetzt waren [86, 149]. Im Bereich der pädiatrischen Onkologie werden bereits ca. 33.000 geheilte ehemalige krebskranke Kinder und Jugendliche in einer Langzeitbeobachtung über das Deutsche Kinderkrebsregister (DKKR) bundesweit erfasst. Langzeitergebnisse 


$\begin{array}{ll}\text { Hoch } & \text { Doxo } \geq 250 \mathrm{mg} / \mathrm{m}^{2} \\ & \mathrm{RT} x \geq 35 \mathrm{~Gy} \\ & \text { Doxo }<250 \mathrm{mg} / \mathrm{m}^{2}+\mathrm{RTx} \geq 15 \mathrm{~Gy} \\ & \text { Alter }<5 \mathrm{~J} \text {. bei Therapie }\end{array}$

Moderat

Doxo $<250 \mathrm{mg} / \mathrm{m}^{2}$

RTx $\geq 15$ Gy bis $<35$ Gy

Nach Therapieabschluss

Optional: 12 Monate nach Therapieende

24 Monate nach Therapieende

5 Jahre nach Therapieende

Alle 5 Jahre

Bei Symptomatik jederzeit

Nach Therapieabschluss

Optional: 12 Monate nach Therapieende

5 Jahre nach Therapieende

Alle 5 Jahre

Bei Symptomatik jederzeit

Weitere Cisplatin Individuell

Substanzen (Hochdosis-)Cyclophosphamid

Mitoxantrone toren

Blutdruck mindestens jährlich

Lipidprofil und $\mathrm{HbA}_{1 \mathrm{c}}$

mindestens alle 3 Jahre,

insbesondere nach RTx

Modifizierbare Risikofaktoren

einstellen:

Nikotin

Gewicht, BMI, WHR

Aufklärung über individuelles

Risikoprofil

Patientenedukation zu

Lebensstil

Doxo Doxorubicin, RTx Bestrahlung, Gy Gray, EKG Elektrokardiogramm, BMI Body-Mass-Index, WHR "waist-to-hip ratio"

liegen für die meisten onkologischen und hämatologischen Therapien noch nicht ausreichend vor, sodass nur eine Empfehlung bei Patienten nach Bestrahlung bzw. stattgehabter AnthrazyklinTherapie abgegeben werden kann.

Auch wenn die Entwicklung neuer schwerer kardialer Funktionsstörungen nach Beendigung einer AnthrazyklinTherapie selten ist und zumindest bei Brustkrebspatientinnen die kardiovaskuläre Mortalität nicht erhöht erscheint [33, 146], wurde die Entwicklung einer Herzinsuffizienz auch viele Jahre nach Exposition mit Anthrazyklinen beobachtet [56, 130, 137]. Ebenso können sich Folgen einer mediastinalen Strahlentherapie erst nach vielen Jahren bzw. Jahrzehnten manifestieren und umfassen Klappenschäden, Veränderungen kleiner und großer Gefäße, Rhythmusstörungen sowie Myokardund Perikarderkrankungen bis hin zur restriktiven Kardiomyopathie [34, 77]. Bei allen Patienten, die potenziell kardiotoxischen Therapien ausgesetzt wurden, sollte daher nach Abschluss der Krebstherapie sowie bei regelmäßigen Verlaufskontrollen eine sorgfältige klinische Untersuchung einschließlich EKG durchgeführt werden (•Tab. 5 und 6). Auf die Notwendigkeit der Kontrolle von kardiovaskulären Risikofaktoren sollte hingewiesen werden sowie eine Sensibilisierung für die Möglichkeit der Entwicklung kardiovaskulärer Folgeer- krankungen und eine Aufklärung über entsprechende Symptome erfolgen.

\section{Nachbeobachtung nach Anthrazyklin-Therapie}

Da bereits geringe Anthrazyklin-Dosen mit einer Herzinsuffizienz oder zumindest subklinischen kardiovaskulären Folgen assoziiert sind und das Risiko für die Entwicklung einer Herzinsuffizienz mit weiteren Risikofaktoren zunimmt [12, 98, 144], sollte auch bei asymptomatischen Patienten nach geringen Anthrazyklin-Dosen im Rahmen der kardiologischen Kontrolluntersuchung eine Echokardiographie durchgeführt werden (- Tab. 5 und 6). Eine Ausnahme erscheint nur bei Patienten vertretbar, die niedrige Anthrazyklin-Dosen im Erwachsenenalter erhalten haben und keine zusätzlichen Risikofaktoren aufweisen (• Tab. 6; [5]). Diese Empfehlungen stehen im Einklang mit einem aktuellen Positionspapier der Arbeitsgemeinschaft „Langzeitbeobachtung“ der Gesellschaft für Pädiatrische Onkologie und Hämatologie (GPOH) [70] und den Empfehlungen der International Late Effects of Childhood Cancer Guideline Harmonization Group [4], welche bei Patienten mit Therapie im Kindes- oder Jugendalter eine Risikostratifizierung in Abhängigkeit der Anthrazyklin-Dosis sowie regelmäßige Echokardiographien und EKGs zur Erkennung einer Kardiomyopathie empfehlen. Zur Risiko- stratifizierung in dieser Patientengruppe kann auch folgendes Online-Tool verwendet werden: https://ccss.stjude.org/ tools-and-documents/calculators-andother-tools/ccss-cardiovascular-riskcalculator.html.

Für die im Erwachsenenalter therapierten Patientinnen und Patienten wurden vielerorts Programme zur kardiologischen Langzeitkontrolle initiiert. Ein Vorschlag zur Langzeitüberwachung nach Anthrazyklin- bzw. TrastuzumabGabe findet sich in $\bullet$ Tab. 6 und basiert auf den Empfehlungen der ASCO [5]. Bei asymptomatischen Patienten sollten initial engmaschige echokardiographische Kontrollen erfolgen aufgrund des in der Regel frühen Neuauftretens kardialer Komplikationen [5, 19]. Im Verlauf sind Echokardiographien seltener erforderlich, wodurch eine unnötige Beunruhigung durch übermäßige Kontrollen vermieden werden kann. Da bei der Echokardiographie neben einer Einschränkung der LVEF auch diastolische Funktionsstörungen, Klappenvitien oder eine Erhöhung des pulmonalarteriellen Druckes gut detektiert werden können, ist eine sorgfältige Untersuchung unabdingbar [110, 149]. StrainAnalysen haben ein hohes Potenzial zukünftig ein echokardiographischer Routineparameter zu werden. Aktuell kann allerdings nur im Einzelfall eine Einbeziehung insbesondere bei longitudinalen Betrachtungen erwogen werden. Alternativ zur Echokardiographie kann 
Tab. 6 Kardiovaskuläre Kontrollen bei asymptomatischen Überlebenden nach Krebstherapie im Erwachsenenalter. (Mod. nach [5, 19, 110])

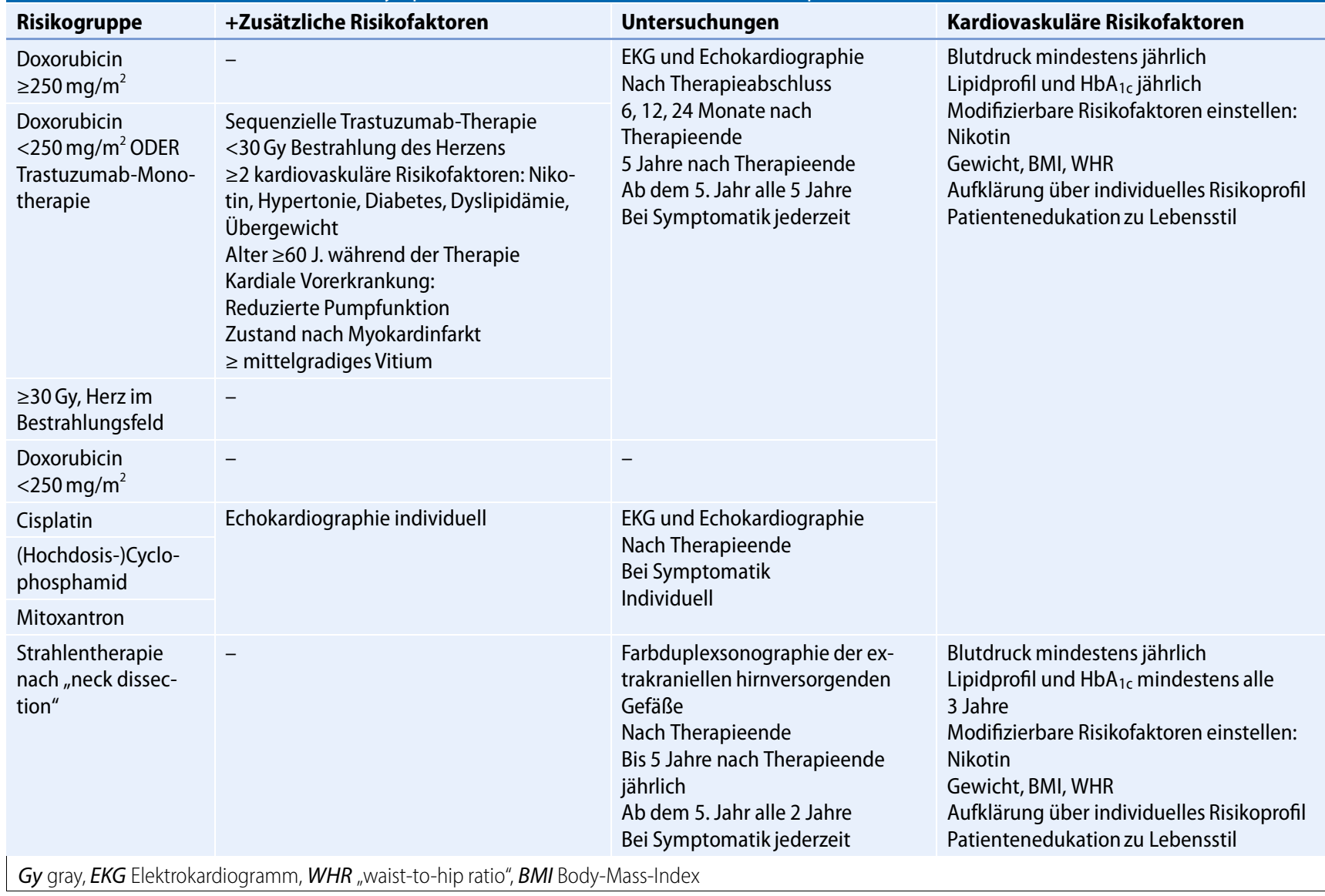

z.B. bei eingeschränkten Schallbedingungen auch ein kardiales MRT erfolgen. Während der Nachweis einer eingeschränkten LVEF die Initiierung einer Herzinsuffizienztherapie auch bei asymptomatischen Patienten zur Folge haben sollte, ist der Stellenwert von Veränderungen im kardialen MRT, wie z. B. ,late Gadolinium enhancement", noch nicht geklärt [82, 117]. Ebenso ist die prognostische Bedeutung von Biomarkern bei asymptomatischen Langzeitüberlebenden einer Krebserkrankung unklar [5]. Somit bedarf es weiterer Studien vor einem Einsatz zum routinemäßigen Screening auf eine Herzinsuffizienz. Bei Nachweis einer Herzinsuffizienz sollte die Therapie basierend auf den ESCEmpfehlungen erfolgen [111], da es für die Einleitung einer Herzinsuffizienztherapie speziell für Langzeitüberlebende einer Krebserkrankung keine breite Datenbasis gibt. In besonderen Situationen, wie z. B. einer Schwangerschaft, sollte bei allen Patientinnen mit vorausgegangener
Anthrazyklin-Therapie eine kardiologische Kontrolle erfolgen, da der erhöhte metabolische Bedarf ggf. zur Progredienz einer subklinischen Herzinsuffizienz führen kann.

\section{Nachbeobachtung bei Radiotherapie}

Auch wenn Fortschritte der Strahlentherapie eine steigende Präzision des Strahlenfeldes ermöglichen, sind mediastinale Bestrahlungen in manchen Fällen unvermeidlich. Strahlenbedingte Sklerosierungen oder Insuffizienzen der Klappen (insbesondere der Aortenklappe) fallen im Rahmen der regelmäßigen echokardiographischen Kontrollen auf, die gemäß einer Empfehlung der European Association of Cardiovascular Imaging/American Society of Echocardiography (EACVI/ASE) dann jährlich im Verlauf kontrolliert werden sollten [68]. Bei asymptomatischen Patienten nach Strahlentherapie, bei der das Herz ein- bezogen ist, ist nach EACVI/ASE ein 5 -jähriges Kontrollintervall ausreichend (- Tab. 5 und 6; [68]).

Vaskuläre Veränderungen können auch außerhalb des Bestrahlungsfeldes auftreten und häufig verborgen bleiben bzw. sich durch eine kardiale Ischämie oder Schlaganfall äußern [25]. Insbesondere bei Langzeitüberlebenden einer zervikalen Strahlentherapie sollten regelmäßige Ultraschallkontrollen der extrakraniellen hirnversorgenden Gefäße erfolgen, um atherosklerotische Veränderungen $\mathrm{zu}$ erkennen, da bei Strahlentherapie von Kopf-Hals-Tumoren die Inzidenz einer Karotisstenose von $18-38 \%$ im Vergleich zu 0-9,2\% bei nicht bestrahlten Patienten zunimmt. Konsequenzen hieraus bestehen in einer intensivierten Kontrolle kardialer Risikofaktoren und bei hochgradiger Karotisstenose auch der Diskussion einer Stentangioplastie oder Operation. 
Tab. 7 Empfehlung zur Implementierung einer onkokardiologischen Struktur auf der Basis des aktuellen Berichtes der Arbeitsgruppe Cardio-oncology der Europäischen Gesellschaft für Kardiologie (ESC) [69]

\begin{tabular}{|c|c|c|c|}
\hline & Basisstruktur/Grundversorger & $\begin{array}{l}\text { Fortgeschrittene Patientenver- } \\
\text { sorgung/Maximalversorgung }\end{array}$ & Spezialisierte Zentren \\
\hline Patientenaufkommen & $<10$ Patienten/Woche & $>10$ Patienten/Woche & $>20$ Patienten/Woche \\
\hline Krankenhausstruktur & $\begin{array}{l}\text { Kardiologische Abteilung } \\
\text { Onkologische Abteilung } \\
\text { Allgemeine Intensivstation }\end{array}$ & $\begin{array}{l}\text { Kardiologische Abteilung } \\
\text { Klinik für Hämatologie/Onkologie/ } \\
\text { Strahlentherapie } \\
\text { Hämatologische Abteilung }\end{array}$ & $\begin{array}{l}\text { Abteilungen wie bei Maximalversor- } \\
\text { ger } \\
\text { Herzinsuffizienzprogramm } \\
\text { Kardiologische Intensivstation }\end{array}$ \\
\hline \multicolumn{4}{|l|}{ Multidisziplinäre Teams } \\
\hline Organisation & $\begin{array}{l}\text { Basis-Onkokardiologieteam oder } \\
\text { spezialisierter Kardiologe } \\
\text { Allgemeine kardiologische Versor- } \\
\text { gung }\end{array}$ & Onkokardiologieteam & $\begin{array}{l}\text { Onkokardiologieteam } \\
\text { Kardiales Rehabilitationszentrum } \\
\text { Heart-failure-Unit } \\
\text { Klappenteam } \\
\text { Forschungsschwerpunkt }\end{array}$ \\
\hline Onkokardiologische Ambulanz & Empfohlen & Vorhanden & Vorhanden \\
\hline $24 / 7$ & Empfohlen & Vorhanden für stationäre Patienten & Vorhanden für stationäre Patienten \\
\hline $\begin{array}{l}\text { Strukturierte klinische Vorge- } \\
\text { hensweisen }\end{array}$ & Vorhanden & Vorhanden & Vorhanden \\
\hline Krebsnachsorgeprogramm & - & Vorhanden & Vorhanden \\
\hline Strukturierte Weiterbildung & - & Implementiert für Personal & $\begin{array}{l}\text { Implementiert für Personal und } \\
\text { Patienten }\end{array}$ \\
\hline \multicolumn{4}{|l|}{ Technische Voraussetzungen } \\
\hline Standardechokardiographie & Vorhanden & Vorhanden & Vorhanden \\
\hline Strain/3-D-Strain & Nicht zwingend & Vorhanden & Vorhanden \\
\hline CMRT, CT & Nicht zwingend & Vorhanden & Vorhanden \\
\hline $\begin{array}{l}\text { Laboruntersuchungen (kardiale } \\
\text { Biomarker) }\end{array}$ & Vorhanden & Vorhanden & Vorhanden/Genetik/neue Biomarker \\
\hline \multicolumn{4}{|l|}{ Prozeduren } \\
\hline $\begin{array}{l}\text { Herzkatheter/ } \\
\text { elektrophysiologische Untersu- } \\
\text { chungen/Herzchirurgie/kardiale } \\
\text { Device-Therapie }\end{array}$ & $\begin{array}{l}\text { Verbund mit größeren, regionalen } \\
\text { Onkokardiologien }\end{array}$ & Vorhanden & $\begin{array}{l}\text { + Versorgung von Patienten mit } \\
\text { terminaler Herzinsuffizienz }\end{array}$ \\
\hline \multicolumn{4}{|l|}{ Datenüberarbeitung } \\
\hline $\begin{array}{l}\text { Datenbanken und Forschungs- } \\
\text { programm }\end{array}$ & Nicht zwingend & Sehr empfohlen & $\begin{array}{l}\text { Implementierter onkokardiologischer } \\
\text { Forschungsschwerpunkt }\end{array}$ \\
\hline cMRT kardiale Magnetresonanztor & graphie, $C T$ Computertomographie & & \\
\hline
\end{tabular}

\section{Onkologische Kardiologie- Teams}

\section{Struktur}

Die Empfehlungen zur Struktur und zum Aufbau einer spezifischen onkokardiologischen Gruppe basieren auf den publizierten Daten aus onkokardiologischen Einrichtungen und den aktuellen Empfehlungen der ESC $[41,69,108]$. Eingebettet ist das onkokardiologische Team in die lokal vorgehaltene kardiologische Struktur und bedient sich deren kardiologischer Diagnostik. Die Größe und Zusammensetzung der onkokardiologischen Teams richtet sich zudem nach den regionalen Bedürfnissen. Entschei- dend sind zum einen die Standortgröße mit dem zu erwartenden Patientenaufkommen, zum anderen die etablierten Schwerpunkte der hämatologischen bzw. onkologischen Krankenversorgung, die einer entsprechenden Anpassung der onkokardiologischen Versorgung bedürfen (• Tab. 7; [28, 69, 108]).

\section{Abstimmung der Patientenwege}

Die diversen onkologischen Therapien basieren auf unterschiedlichen zeitlichen Abfolgen, denen sich die onkokardiologische Mitbetreuung anpassen muss. Dies ist entsprechend den lokalen Vorbedingungen $\mathrm{zu}$ adaptieren und kann einen Schwerpunkt im ambulanten und/oder stationären Bereich bilden. Eine Vorstellung von Patienten in der Onkokardiologie sollte bei bestimmten Patientengruppen bzw. geplanten Therapien in Abstimmung mit dem behandelnden Onkologen als standardisiertes Vorgehen etabliert werden [149].

\section{Basisstruktur}

Grundsätzlich sollte die Voraussetzung zur Anamneseerhebung, zu der Durchführung einer körperlichen Untersuchung sowie eines EKGs vorhanden sein [69]. An bildgebender Diagnostik sollte die Durchführbarkeit einer Echokardiographie mit Strain-Analyse (ggf. 3-D-Strain) als Basisbetreuung onkolo- 
gischer Patienten geschaffen sein [110]. Eine Labordiagnostik, insbesondere zur Bestimmung kardialer Serumparameter (Troponin, BNP, NT-proBNP), sollte zur Verfügung stehen. Eine weiterführende kardiologische Diagnostik erfolgt im regionalen Verbund mit größeren Krankenhäusern oder spezialisierten Einrichtungen (beispielsweise eine universitäre Sektion Onkokardiologie). Apparative onkokardiologische Basisversorgung beinhaltet demnach:

- EKG,

- 24-h-EKG,

- Echokardiographie ggf. 3-D-LVEF, Strain und ggf. auch 3-D-Strain,

- Labordiagnostik einschließlich kardialer Serumparameter.

\section{Zentrumsstrukturen (Maximalver- sorger)}

Eine weiterführende kardiale Diagnostik erfordert die diagnostischen und therapeutischen Voraussetzungen, wie sie in einem Haus der kardiologischen Maximalversorgung vorliegen. Hierzu gehören neben den Gegebenheiten der Basisversorgung Onkokardiologie:

- kardiales MRT,

- CT,

- Rechtsherzkatheter,

- Linksherzkatheter mit Möglichkeit zur Koronarintervention und Myokardbiopsie,

- PET-CT (bisher nur in Studien),

- ggf. Möglichkeiten zur diagnostischen und therapeutischen elektrophysiologischen Untersuchung (EPU),

- Device-Therapien.

Um interdisziplinäre onkokardiologische Fragestellungen $\mathrm{zu}$ beantworten, bedarf es einer regelmäßigen Abstimmung mit dem behandelnden Hämatologen bzw. Onkologen. Dies kann konsiliarisch im Rahmen bereits bestehender TumorBoards erfolgen oder unabhängig davon, beispielsweise im Rahmen regelmäßig stattfindender onkokardiologischer Konferenzen.

Zur standardisierten Wiedervorstellung von Patienten (beispielsweise nach neoadjuvanter zytostatischer Therapie oder kurzfristig nach Initialisierung einer Immuncheckpoint-Inhibitor-Therapie) sollten interne Abläufe gemäß dem aktuellen Positionspapier sowie dem Positionspapier der ESC festgelegt werden [149].

Bei spezialisierten sind ist zudem eine Weiterbildung von ärztlichem und nichtärztlichem Personal (beispielsweise als Teil eines Heart-Nurse-Kurses) sowie strukturierte Informationsweitergabe an Patienten (beispielsweise Informationsabende) empfohlen.

Bei sehr hohem Patientenaufkommen kann ein spezifischer onkokardiologischer Konsildienst etabliert werden, der im Rahmen bereits erhobener kardialer Befunde eine beratende Funktion übernimmt.

\section{Ausbildung}

Im Curriculum „Allgemeine Kardiologie“ sind die Punkte zur onkokardiologischen Ausbildung im Abschnitt „Beruflich-professionelles Verhalten 12“ wie folgt hinterlegt: Beratung des Onkologen hinsichtlich möglicher Einschränkungen der geplanten Tumorbehandlung aufgrund vorbestehender Herzerkrankungen sowie in den theoretischen Kenntnissen: Kenntnisse der Nebenwirkungen der medikamentösen, strahlentherapeutischen und chirurgischen Behandlung am Herzen. Als praktische Fertigkeiten ist Level III im Bereich Echokardiographie gefordert (höchste Ausbildungsstufe).

Die Ausbildung zur spezifischen onkokardiologischen Diagnostik und Therapie erfolgt im Rahmen der kardiologischen Weiterbildung und ist in wesentlichen Teilen dort implementiert.

Eine Rotation auf eine Station mit hämatologischem oder onkologischem Schwerpunkt im Rahmen der kardiologischen Facharztausbildung ist für eine spätere, weiterführende onkokardiologische Mitbetreuung von Patienten bei Spezialisierung in onkokardiologischen Sektionen/Teams wünschenswert.

Essenziell sind die Beurteilung des kardiologischen Risikoprofils für kardiovaskuläre Erkrankungen sowie die Diagnostik und Therapie kardialer Komplikationen während bzw. nach onkologischer Therapie.
Dies beinhaltet:

- Einschränkung der LVEF,

- KHK,

- Arrhythmien,

- arterielle Hypertonie,

- pAVK,

- pulmonalarterielle Hypertonie,

- Myokarditis,

- Perikarditis.

Die aufgelisteten Erkrankungen können sowohl als Folge der onkologischen Therapie als auch eines überlappenden Risikoprofils auftreten.

Es ist zudem sinnvoll, eine Einschätzung des spezifischen kardialen Risikos bestimmter onkologischer Therapien (beispielsweise die Diagnostik und Therapie von Immuncheckpoint-Inhibitorinduzierter Myokarditis oder die Diagnostik und Therapie der pulmonalen Hypertonie bei der Verwendung von Alkylanzien) im Rahmen einer strukturierten Weiterbildung gesondert $\mathrm{zu}$ behandeln $[69,106,113,120]$.

Als Teile einer strukturierten Weiterbildung sollten zudem Basiskenntnisse auf dem Gebiet der Onkologie und Hämatologie vermittelt werden, v. a. Grundlagen zur Therapieentscheidung und $\mathrm{zu}$ Therapiemöglichkeiten sowie die genaue Nomenklatur [69].

Eine Forderung nach einer spezialisierten Weiterbildung Onkokardiologie mit verschiedenen Ausbildungszeiten und -inhalten (Level I-III) ist von der internationalen Gesellschaft für Kardioonkologie und der kanadischen Gesellschaft für onkologische Kardiologie in einem gemeinsamen Positionspapier empfohlen worden [78]. Eine Empfehlung der Deutschen Gesellschaft für Kardiologie wird sich nach den europäischen Empfehlungen richten, die gegenwärtig in der ESC erarbeitet werden.

\section{Patientenauswahl}

Die Auswahl von Patienten für eine onkokardiologische Mitbetreuung (s. • Tab. 8) erfolgt im Prinzip in 3 Gruppen:

\section{Patienten vor einer geplanten onkologischen Therapie}

Eine kardiologische Mitbetreuung onkologischer Patienten vor einer onkologi- 
Tab. 8 Patientenauswahl in der Onkokardiologie, adaptiert vom aktuellen Positionspapier der Europäischen Gesellschaft für Kardiologie (ESC) [69]

Vor onkologischer Therapie

Identifizierung von Patienten mit ho-

hem kardiovaskulärem Risiko

Identifizierung von Therapien mit hohem kardialem Risiko

Aufgabe des Onkokardiolo-

gen

Weiterführende Diagnostik bzw. Initialisierung einer Therapie. Beratung des Onkologen
Während onkologischer Therapie

Monitoring von Patienten mit hohem

kardiovaskulärem Risiko

Monitoring von Patienten mit onkologischen Therapien mit hohem kardialem Risiko

Behandlung von Patienten mit erhöhten kardialen Biomarkern oder kardialer Symptomatik

Diagnostik und Therapie pathologischer kardialer Befunde
Nach onkologischer Therapie

Kardiologische Betreuung von Patienten nach potenziell kardiotoxischer Therapie

Initialisierung einer kardiologischen Therapie bzw. Einleitung weiterführender kardialer Diagnostik schen Therapie ist notwendig bei Patienten mit einem hohen kardiovaskulären Risiko oder bei einer geplanten Therapie, die mit einem hohen Risiko für eine kardiovaskuläre Nebenwirkung verbunden ist.

$\mathrm{Zu}$ den Therapien mit einem zu hohen Risiko für eine kardiovaskuläre Komplikation gehören:

- Anthrazykline,

- Immuncheckpoint-Inhibitoren,

- BRAF-Inhibitoren,

- Proteasominhibitoren,

- Alkylanzien,

- Bruton-Tyrosinkinase-Inhibitoren.

Basierend auf den Ergebnissen ist eine interdisziplinäre Besprechung der Befunde zur optimierten Versorgung und ggf. Beratung des behandelnden Onkologen zur Therapieentscheidung notwendig.

\section{Patienten während einer onkologischen Therapie}

Eine onkokardiologische Mitbeurteilung während einer onkologischen Therapie ist bei oben genannten onkologischen Therapien mit einem erhöhten Risiko für eine kardiovaskuläre Nebenwirkung notwendig. Unabhängig davon werden Patienten unter einer onkologischen Therapie mit dem Auftreten kardialer Symptome bzw. einer Erhöhung kardialer Biomarker (onko)kardiologisch betreut bzw. die auftretenden kardiologischen Befunde konsiliarisch mitbeurteilt.

Hierzu gehören auch interdisziplinäre Fragestellungen zur Festlegung oder Weiterführung der onkologischen Therapie zugunsten einer optimalen onkologischen Versorgung. Wichtiger Aspekt in diesem Patientenkollektiv ist der
Status der onkologischen Grunderkrankung (palliativ, neoadjuvant, adjuvant). Hieraus ergeben sich auch Konsequenzen für kardiologische Entscheidungen, die beispielsweise von der erwarteten Überlebenszeit der Patienten abhängen und eine interdisziplinäre Abwägung mit der zur erwartenden Verbesserung der Lebensqualität benötigen. In die Entscheidungsprozesse sind die Patienten eng einzubeziehen.

\section{Patienten nach stattgehabter onkologischer Therapie}

Die Datenlage für eine Betreuung nach einer stattgehabten onkologischen Therapie ist gering. Zudem sind die vorliegenden Studien zum Auftreten kardiovaskulärer Komplikationen nach einer onkologischen Erkrankung und Therapie überwiegend retrospektiv durchgeführt worden. Eine Empfehlung zur Betreuung dieses Patientenkollektivs basiert daher auf den aktuellen Positionspapieren der ESC [149]. Generell ist es Aufgabe der Onkokardiologie, onkologische Patienten nach erfolgreicher Therapie über ein erhöhtes kardiovaskuläres Risiko aufzuklären [69]. Zudem sollten strukturierte Nachsorgeprogramme etabliert werden für Patienten mit hohem Risiko (beispielsweise nach Bestrahlung im herznahen Thoraxbereich oder nach stattgehabter Anthrazyklin-Therapie).

\section{Korrespondenzadresse}

Univ.-Prof. Dr. med. Tienush Rassaf

Klinik für Kardiologie und Angiologie, Westdeutsches Herz- und Gefäßzentrum, Universitätsklinikum Essen Hufelandstr. 55, 45147 Essen, Deutschland tienush.rassaf@uk-essen.de

\section{Einhaltung ethischer Richtlinien}

Interessenkonflikt. Den Interessenkonflikt der Autoren finden Sie online auf der DGK-Homepage unter http://leitlinien.dgk.org/bei der entsprechenden Publikation.

Für diesen Beitrag wurden von den Autoren keine Studien an Menschen oder Tieren durchgeführt. Für die aufgeführten Studien gelten die jeweils dort angegebenen ethischen Richtlinien.

\section{Literatur}

1. Abdel-Rahman O (2019) 5-fluorouracil-related cardiotoxicity; findings from five randomized studies of 5-fluorouracil-based regimens in metastatic colorectal cancer. Clin Colorectal Cancer 18:58-63

2. Agewall S, Giannitsis E, Jernberg T et al (2011) Troponin elevation in coronary vs. non-coronary disease. Eur Heart J 32:404-411

3. Anquetil C, Salem JE, Lebrun-Vignes B et al (2018) Immune checkpoint inhibitor-associated myositis. Circulation 138:743-745

4. Armenian SH, Hudson MM, Mulder RL et al (2015) Recommendations for cardiomyopathy surveillance for survivors of childhood cancer: a report from the International Late Effects of Childhood Cancer Guideline Harmonization Group. Lancet Oncol 16:e123-e136

5. Armenian SH, Lacchetti C, Barac A et al (2017) Prevention and monitoring of cardiac dysfunction in survivors of adult cancers: American Society of Clinical Oncology clinical practice guideline. J Clin Oncol 35:893-911

6. Armenian SH, Sun CL, Francisco L et al (2008) Late congestive heart failure after hematopoietic cell transplantation.J Clin Oncol 26:5537-5543 
7. Armenian SH, Xu L, Ky B et al (2016) Cardiovascular disease among survivors of adult-onset cancer: a community-based retrospective cohort study. JClin Oncol 34:1122-1130

8. Armstrong GT, Joshi VM, Ness KK et al (2015) Comprehensive echocardiographic detection of treatment-related cardiac dysfunction in adult survivors of childhood cancer: results from the st. Jude lifetime cohort study. J Am Coll Cardiol 65:2511-2522

9. Atkins KM, Rawal B, Chaunzwa TL et al (2019) Cardiac radiation dose, cardiac disease, and mortality in patients with lung cancer. J Am Coll Cardiol 73:2976

10. Barac A, Blaes A, Lynce F (2019) Lessons from primary cardiac prevention trials during trastuzumab therapy: end of one size fits all. J Am Coll Cardiol 73:2869-2871

11. Bhatia S (2011) Role of genetic susceptibility in development of treatment-related adverse outcomes in cancer survivors. Cancer Epidemiol Biomarkers Prev 20:2048-2067

12. Bowles EJ, Wellman R, Feigelson HS et al (2012) Risk of heart failure in breast cancer patients after anthracycline and trastuzumab treatment: a retrospective cohort study. J Natl Cancer Inst 104:1293-1305

13. Brahmer JR, Lacchetti C, Schneider BJ et al (2018) Management of immune-related adverse events in patients treated with immune checkpoint inhibitor therapy: American Society of Clinical Oncology clinical practice guideline. J Clin Oncol 36:1714-1768

14. Braumann S, Peitsch WK, Pfister R et al (2019) Angina pectoris in a 47-year-old athletic man with psoriasis vulgaris. Internist 60:86-89

15. Burridge PW, Li YF, Matsa E et al (2016) Human induced pluripotent stem cell-derived cardiomyocytes recapitulate the predilection of breast cancer patients to doxorubicin-induced cardiotoxicity. Nat Med 22:547-556

16. Caforio AL, Pankuweit S, Arbustini E et al (2013) Current state of knowledge on aetiology, diagnosis, management, and therapy of myocarditis: a position statement of the European Society of Cardiology Working Group on Myocardial and Pericardial Diseases. Eur Heart J 34:2636-2648 (2648a-2648d)

17. Calvillo-Arguelles O, Abdel-Qadir H, Michalowska M et al (2019) Cardioprotective effect of Statins in patients with HER2-positive breast cancer receiving trastuzumab therapy. Can J Cardiol 35:153-159

18. Caocci G, Mulas O, Bonifacio M et al (2019) Recurrent arterial occlusive events in patients with chronic myeloid leukemia treated with secondand third-generation tyrosine kinase inhibitors and role of secondary prevention. Int J Cardiol 288:124-127

19. Cardinale D, Colombo A, Bacchiani G et al (2015) Early detection of anthracycline cardiotoxicity and improvement with heart failure therapy. Circulation 131:1981-1988

20. Centanni M, Moes D, Troconiz IF et al (2019) Clinical pharmacokinetics and pharmacodynamics of immune checkpoint inhibitors. Clin Pharmacokinet 58:835-857

21. Chang HM, Okwuosa TM, Scarabelli T et al (2017) Cardiovascular complications of cancer therapy: best practices in diagnosis, prevention, and management: part 2. J Am Coll Cardiol 70:2552-2565

22. Chari A, Stewart AK, Russell SD et al (2018) Analysis of carfilzomib cardiovascular safety profile across relapsed and/or refractory multiple myeloma clinical trials. Blood Adv 2:1633-1644

23. Chen Y, Chow EJ, Oeffinger KC et al (2019) Traditional cardiovascular risk factors and individual prediction of cardiovascular events in childhood cancer survivors. J Natl Cancer Inst 112:256. https:// doi.org/10.1093/jnci/djz108

24. Chow EJ, Baker KS, Lee SJ et al (2014) Influence of conventional cardiovascular risk factors and lifestyle characteristics on cardiovascular disease after hematopoietic cell transplantation. J Clin Oncol 32:191-198

25. ChowEJ,Chen Y,Hudson MMetal (2018)Prediction of ischemic heart disease and stroke in survivors of childhood cancer. JClin Oncol 36:44-52

26. Cornell RF, Ky B, Weiss BM et al (2019) Prospective study of cardiac events during proteasome inhibitor therapy for relapsed multiple myeloma. JClin Oncol 37:1946-1955

27. Cortes JE, KimDW, Pinilla-lbarz Jetal(2013) A phase 2 trial of ponatinib in Philadelphia chromosomepositive leukemias. N Engl J Med 369:1783-1796

28. Cubbon RM, Lyon AR (2016) Cardio-oncology: concepts and practice. Indian Heart J 68(Suppl 1):S77-S85

29. Cuomo JR, Sharma GK, CongerPDetal (2016) Novel concepts in radiation-induced cardiovascular disease. World J Cardiol 8:504-519

30. Cutter DJ, Schaapveld M, Darby SC et al (2015) Risk of valvular heart disease after treatment for Hodgkin lymphoma. J Natl Cancer Inst. https://doi. org/10.1093/jnci/djv008

31. Danhof S, Schreder M, Rasche L et al (2016) 'Reallife' experience of preapproval carfilzomib-based therapy in myeloma-analysis of cardiac toxicity and predisposing factors. Eur J Haematol 97:25-32

32. Darby SC, Ewertz M, Mcgale P et al (2013) Risk of ischemic heart disease in women after radiotherapy for breast cancer. $\mathrm{N}$ Engl J Med 368:987-998

33. De Azambuja E, Ameye L, Diaz M et al (2015) Cardiac assessment of early breast cancer patients 18 years after treatment with cyclophosphamidemethotrexate-, fluorouracil- or epirubicin-based chemotherapy. Eur JCancer 51:2517-2524

34. Desai MY, Windecker S, Lancellotti P et al (2019) Prevention, diagnosis, and management of radiation-associated cardiac disease: JACC scientific expert panel. J Am Coll Cardiol 74:905-927

35. Dimopoulos MA, Chen C, Spencer A et al (2009) Long-term follow-up on overall survival from the MM-009 and MM-010 phase III trials of lenalidomide plus dexamethasone in patients with relapsed or refractory multiple myeloma. Leukemia 23:2147-2152

36. Dimopoulos MA, Moreau P, Palumbo A et al (2016) Carfilzomib and dexamethasone versus bortezomib and dexamethasone for patients with relapsed or refractory multiple myeloma (ENDEAVOR): a randomised, phase 3, open-label, multicentre study. Lancet Oncol 17:27-38

37. Dobbin SJH, Cameron AC, Petrie MC et al (2018) Toxicity of cancer therapy: what the cardiologist needs to know about angiogenesis inhibitors. Heart 104:1995-2002

38. Doherty KR, Wappel RL, Talbert DR et al (2013) Multi-parameter in vitro toxicity testing of crizotinib, sunitinib, erlotinib, and nilotinib in human cardiomyocytes. Toxicol Appl Pharmacol 272:245-255

39. Escudier M, Cautela J, Malissen Netal (2017)Clinical features, management, and outcomes of immune checkpoint inhibitor-related cardiotoxicity. Circulation 136:2085-2087
40. Fahdi IE, Gaddam V, Saucedo JF et al (2004) Bradycardia during therapy for multiple myeloma with thalidomide. Am J Cardiol 93:1052-1055

41. Fiuza M, Ribeiro L, Magalhaes A et al (2016) Organization and implementation of a cardiooncology program. Rev Port Cardiol 35:485-494

42. Fradley MG, Groarke JD, Laubach J et al (2018) Recurrent cardiotoxicity potentiated by the interaction of proteasome inhibitor and immunomodulatory therapy for the treatment of multiple myeloma. Br J Haematol 180:271-275

43. Ganatra S, Sharma A, Shah S et al (2018) Ibrutinib-associated atrial fibrillation. JACC Clin Electrophysiol 4:1491-1500

44. Gandini S, Puntoni M, Heckman-Stoddard BM et al (2014) Metformin and cancer risk and mortality: a systematic review and meta-analysis taking into account biases and confounders. Cancer Prev Res 7:867-885

45. Ghatalia P, Je Y, Kaymakcalan MD et al (2015) QTC interval prolongation with vascular endothelial growth factor receptor tyrosine kinase inhibitors. Br JCancer 112:296-305

46. Ghatalia P, Morgan CJ, Je Y et al (2015) Congestive heart failure with vascular endothelial growth factor receptor tyrosine kinase inhibitors. Crit Rev Oncol Hematol 94:228-237

47. Giovannucci E, Harlan DM, Archer MC et al (2010) Diabetes and cancer: a consensus report. Diabetes Care 33:1674-1685

48. Goel S, Liu J, Guo H et al (2019) Decline in left ventricular ejection fraction following anthracyclines predicts trastuzumab cardiotoxicity. JACC Heart Fail 7:795-804

49. Groarke JD, Nguyen PL, Nohria A et al (2014) Cardiovascular complications of radiation therapy for thoracic malignancies: the role for non-invasive imaging for detection of cardiovascular disease. Eur Heart J35:612-623

50. Guglin M, Krischer J, Tamura R et al (2019) Randomized trial of lisinopril versus carvedilol to prevent trastuzumab cardiotoxicity in patients with breast cancer. J Am Coll Cardiol 73:2859-2868

51. Guignabert C, Phan C, Seferian A et al (2016) Dasatinib induces lung vascular toxicity and predisposes to pulmonary hypertension. J Clin Invest 126:3207-3218

52. Haas NB, Manola J, Ky B et al (2015) Effects of adjuvant sorafenib and sunitinib on cardiac function in renal cell carcinoma patients without overtmetastases: results from ASSURE, ECOG 2805. Clin Cancer Res 21:4048-4054

53. Haddy N, Diallo S, El-Fayech C et al (2016) Cardiac diseases following childhood cancer treatment. Circulation 133:31-38

54. Hamadi A, Grigg AP, Dobie G et al (2019) Ponatinib tyrosine kinase inhibitor induces a thromboinflammatory response. Thromb Haemost 119:1112-1123

55. Heckmann MB, Doroudgar S, Katus HA et al (2018) Cardiovascular adverse events in multiple myeloma patients. JThorac Dis 10:S4296-S4305

56. Hequet O, Le QH, Moullet I et al (2004) Subclinical late cardiomyopathy after doxorubicin therapy for lymphoma in adults. JClin Oncol 22:1864-1871

57. Hilfiker-Kleiner $D$, Ardehali $H$, Fischmeister $R$ et al (2019) Late onset heart failure after childhood chemotherapy. Eur Heart J 40:798-800

58. Hooning MJ, Botma A, Aleman BMP et al (2007) Long-term risk of cardiovascular disease in 10year survivors of breast cancer. J Natl Cancer Inst 99:365-375 
59. Hu JR, Florido R, Lipson EJ et al (2019) Cardiovascular toxicities associated with immune checkpoint inhibitors. Cardiovasc Res 115:854-868

60. Jaworski C, Mariani JA, Wheeler G et al (2013) Cardiac complications of thoracic irradiation. J Am Coll Cardiol 61:2319-2328

61. Jerusalem G, Lancellotti P, Kim SB (2019) HER2+ breast cancer treatment and cardiotoxicity: monitoring and management. Breast Cancer Res Treat 177:237-250

62. Jiang L, Li L, Ruan Y et al (2019) Ibrutinib promotes atrial fibrillation by inducing structural remodeling and calcium dysregulation in the atrium. Heart Rhythm 16:1374-1382

63. John S, Antonia SJ, Rose TA et al (2017) Progressive hypoventilation due to mixed CD8(+) and CD4(+) lymphocytic polymyositis following tremelimumab-durvalumab treatment. J Immunother Cancer 5:54

64. Johnson DB, Balko JM, Compton ML et al (2016) Fulminant myocarditis with combination immune checkpoint blockade. NEnglJMed 375:1749-1755

65. Kloth JS, Pagani A, Verboom MC et al (2015) Incidence and relevance of QTc-interval prolongation caused by tyrosine kinase inhibitors. Br J Cancer 112:1011-1016

66. Knight R, Delap RJ, Zeldis JB (2006) Lenalidomide and venous thrombosis in multiple myeloma. NEngl J Med 354:2079-2080

67. Kosalka P, Johnson C, Turek M et al (2019) Effect of obesity, dyslipidemia, and diabetes on trastuzumab-related cardiotoxicity in breast cancer. Curr Oncol 26:e314-e321

68. Lancellotti P, Nkomo VT, Badano LP et al (2013) Expert consensus for multi-modality imaging evaluation of cardiovascular complications of radiotherapy in adults: a report from the European Association of Cardiovascular Imaging and the American Society of Echocardiography. Eur Heart Cardiovasc Imaging 14:721-740

69. Lancellotti P, Suter TM, Lopez-Fernandez T et al (2019) Cardio-oncology services: rationale, organization, and implementation. Eur Heart J 40:1756-1763

70. Langer T, Grabow D, Kaatsch P et al (2018of) Long-Term Follow-Up in Childhood Cancer Survivors-Position paper 2018 of the working group "long-term follow-up" of the Society of Pediatric Oncology and Hematology (GPOH) on long-term surveillance, long-term follow-up and late effect evaluation in pediatric oncology patients. Klin Padiatr 230:291-298

71. Larson RA, Kim D-W, Issaragrilsil S et al (2014) Efficacy and safety of nilotinib (NIL) vs imatinib (IM) in patients (pts) with newly diagnosed chronic myeloid leukemia in chronic phase (CML-CP): long-term follow-up $(\mathrm{f} / \mathrm{u})$ of ENESTnd. Blood 124:4541-4541

72. Latifi Y, Moccetti F, Wu M et al (2019) Thrombotic microangiopathy as a cause of cardiovascular toxicity from the BCR-ABL1 tyrosine kinase inhibitor ponatinib. Blood 133:1597-1606

73. Chuy LK, Nahhas O, Dominic P et al (2019) Cardiovascular complications associated with mediastinal radiation. Curr Treat Options Cardiovasc Med 21:31

74. Lee DH, Fradley MG (2018) Cardiovascular complications of multiple myeloma treatment: evaluation, management, and prevention. Curr Treat Options Cardiovasc Med 20:19

75. Lee HA, Hyun SA, Byun B et al (2018) Electrophysiological mechanisms of vandetanib-induced cardiotoxicity: comparison of action potentials in rabbit Purkinje fibers and pluripotent stem cellderived cardiomyocytes. PLoSONE 13:e195577

76. Lehmann LH, Worst BC, Stanmore DA et al (2014) Histone deacetylase signaling in cardioprotection. Cell Mol Life Sci 71:1673-1690

77. Lenihan DJ, Cardinale DM (2012) Late cardiac effects of cancer treatment. J Clin Oncol 30:3657-3664

78. Lenihan DJ, Hartlage G, Decara Jet al (2016) Cardio oncology training: a proposal from the international cardioncology society and Canadian Cardiac Oncology network for a new multidisciplinary specialty. J Card Fail 22:465-471

79. Leonard JT, Rowley JS, Eide CA etal (2016) Targeting BCL-2 and ABL/LYN in Philadelphia chromosomepositive acute lymphoblastic leukemia. Sci Transl Med 8:354ra114

80. Lip GY, Nieuwlaat R, Pisters R et al (2010) Refining clinical risk stratification for predicting stroke and thromboembolism in atrial fibrillation using a novel risk factor-based approach: the euro heart survey on atrial fibrillation. Chest 137:263-272

81. Lipshultz SE, Adams MJ, Colan SD et al (2013) Long-term cardiovascular toxicity in children, adolescents, and young adults who receive cancer therapy: pathophysiology, course, monitoring, management, prevention, and research directions: a scientific statement from the American Heart Association. Circulation 128:1927-1995

82. Lustberg MB, Reinbolt $R$, Addison $D$ et al (2019) Early detection of anthracycline-induced cardiotoxicity in breast cancer survivors with T2 cardiac magnetic resonance. Circ Cardiovasc Imaging 12:e8777

83. Lynce F, Barac A, Geng X et al (2019) Prospective evaluation of the cardiac safety of HER2-targeted therapies in patients with HER2-positive breast cancer and compromised heart function: the SAFE HEaRt study. Breast Cancer Res Treat 175:595-603

84. Lyon AR, YousafN, Battisti NMLetal (2018) Immune checkpoint inhibitors and cardiovascular toxicity. Lancet Oncol 19:e447-e458

85. Mahmood SS, Fradley MG, Cohen JV et al (2018) Myocarditis in patients treated with immune checkpoint inhibitors. J Am Coll Cardiol 71:1755-1764

86. Mccabe MS, Bhatia S, Oeffinger KC et al (2013) American society of clinical oncology statement: achieving high-quality cancer survivorship care. JClin Oncol 31:631-640

87. Mclaughlin PY, Kong W, De Metz C et al (2018) Do radiation oncology outreach clinics affect the use of radiotherapy? Radiother Oncol 127:143-149

88. Medeiros BC, Possick J, Fradley M (2018) Cardiovascular, pulmonary, and metabolic toxicities complicating tyrosine kinase inhibitor therapy in chronic myeloid leukemia: Strategies for monitoring, detecting, and managing. Blood Rev 32:289-299

89. Meid AD, Bighelli I, Machler S et al (2017) Combinations of QTc-prolonging drugs: towards disentangling pharmacokinetic and pharmacodynamic effects in their potentially additive nature. Ther Adv Psychopharmacol 7:251-264

90. Meijers WC, De Boer RA (2019) Common risk factors for heart failure and cancer. Cardiovasc Res 115:844-853

91. Michel L, Mincu RI, Mahabadi AA et al (2019) Troponins and brain natriuretic peptides for the prediction of cardiotoxicity in cancer patients: a meta-analysis. Eur JHeart Fail. https://doi.org/10. 1002/ejhf.1631

92. Michel L, Rassaf T, Totzeck M (2018) Biomarkers for the detection of apparent and subclinical cancer therapy-related cardiotoxicity. J Thorac Dis 10:S4282-S4295

93. Mincu RI, Mahabadi AA, Michel L et al (2019) Cardiovascular adverse events associated with BRAF and MEK inhibitors: a systematic review and meta-analysis. Jama Netw Open 2:e198890

94. Moller TR, Garwicz S, Barlow L et al (2001) Decreasing late mortality among five-year survivors of cancer in childhood and adolescence: a population-based study in the Nordic countries.J Clin Oncol 19:3173-3181

95. Moreau P, San Miguel J, Sonneveld P et al (2017) Multiple myeloma: ESMO Clinical Practice Guidelines for diagnosis, treatment and follow-up Ann Oncol 28:iv52-iv61

96. Moslehi JJ, Johnson DB, Sosman JA (2017) Myocarditis with immune checkpoint blockade. NEngl J Med 376:292

97. Moslehi JJ, Salem JE, Sosman JA et al (2018) Increased reporting of fatal immune checkpoint inhibitor-associated myocarditis. Lancet 391:933

98. Mulrooney DA, Yeazel MW, Kawashima T et al (2009) Cardiac outcomes in a cohort of adult survivors of childhood and adolescent cancer: retrospective analysis of the Childhood Cancer Survivor Study cohort. BMJ 339:b4606

99. Murphy KT (2016) The pathogenesis and treatment of cardiac atrophy in cancer cachexia. Am J Physiol HeartCirc Physiol 310:H466-H477

100. Narayan V, Keefe S, Haas N et al (2017) Prospective evaluation of sunitinib-induced cardiotoxicity in patients with metastatic renal cell carcinoma. Clin Cancer Res 23:3601-3609

101. Nelson ER, Wardell SE, Jasper JS et al (2013) $27-$ Hydroxycholesterol links hypercholesterolemia and breast cancer pathophysiology. Science 342:1094-1098

102. Nhola LF, Abdelmoneim SS, Villarraga HR et al (2019) Echocardiographic assessment for the detection of cardiotoxicity Due to vascular endothelial growth factor inhibitor therapy in metastatic renal cell and colorectal cancers. J Am SocEchocardiogr 32:267-276

103. Nowis D, Maczewski M, Mackiewicz U et al (2010) Cardiotoxicity of the anticancer therapeutic agent bortezomib. Am J Pathol 176:2658-2668

104. Nowsheen S, Aziz K, Park JY et al (2018) Trastuzumab in female breast cancer patients with reduced left ventricular ejection fraction. J Am Heart Assoc 7:e8637

105. Oeffinger KC, Mertens AC, Sklar CA et al (2006) Chronic health conditions in adult survivors of childhood cancer. NEngl J Med 355:1572-1582

106. Okwuosa TM, Akhter N, Williams KA et al (2015) Building a cardio-oncology program in a smallto medium-sized, nonprimary cancer center, academic hospital in the USA: challenges and pitfalls. Future Cardiol 11:413-420

107. Ou SH, Tang Y, Polli A et al (2016) Factors associated with sinus bradycardia during crizotinib treatment: a retrospective analysis of two largescale multinational trials (PROFILE 1005 and 1007). Cancer Med 5:617-622

108. Parent S, Pituskin E, Paterson DI (2016) The cardiooncology program: a multidisciplinary approach to the care of cancer patients with cardiovascular disease. Can J Cardiol 32:847-851

109. Pfeffer TJ, Schlothauer S, Pietzsch S et al (2019) Increased cancer prevalence in peripartum cardiomyopathy. J Am Coll Cardiol CardioOncol. https://doi.org/10.1016/j.jaccao.2019.09.008

110. Plana JC, Galderisi M, Barac A et al (2014) Expert consensus for multimodality imaging evaluation of adult patients during and after 
cancer therapy: a report from the American Society of Echocardiography and the European Association of Cardiovascular Imaging. J Am Soc Echocardiogr 27:911-939

111. Ponikowski P, Voors AA, Anker SD et al (2016) 2016 ESC Guidelines for the diagnosis and treatment of acute and chronic heart failure: The Task Force for the diagnosis and treatment of acute and chronic heart failure of the European Society of Cardiology (ESC) developed with the special contribution of the Heart Failure Association (HFA) of the ESC. Eur Heart J 37:2129-2200

112. Qazilbash MH, Amjad Al, Qureshi S et al (2009) Outcome of allogeneic hematopoietic stem cell transplantation in patients with low left ventricular ejection fraction. Biol Blood Marrow Transplant 15:1265-1270

113. Ranchoux B, Gunther S, Quarck R et al (2015) Chemotherapy-induced pulmonary hypertension: role of alkylating agents. Am J Pathol 185:356-371

114. Rea D, Mirault T, Raffoux E et al (2015) Usefulness of the 2012 European CVD risk assessment model to identify patients at high risk of cardiovascular events during nilotinib therapy in chronic myeloid leukemia. Leukemia 29:1206-1209

115. Reneau JC, Asante D, Van Houten $\mathrm{H}$ et al (2017) Cardiotoxicity risk with bortezomib versus lenalidomide for treatment of multiple myeloma: a propensity matched study of 1,790 patients. Am J Hematol 92:E15-E17

116. Ricke-Hoch M, Hoes MF, Pfeffer TJ et al (2019) In peripartum cardiomyopathy plasminogen activator inhibitor-1 is a potential new biomarker with controversial roles. Cardiovasc Res. https:// doi.org/10.1093/cvr/cvz300

117. Rodriguez-Veiga $R$, Igual $B$, Montesinos $P$ et al (2017) Assessment of late cardiomyopathy by magnetic resonance imaging in patients with acute promyelocytic leukaemia treated with alltrans retinoic acid and idarubicin. Ann Hemato 96:1077-1084

118. Rosenthal A, Luthi J, Belohlavek M et al (2016) Carfilzomib and the cardiorenal system in myeloma: an endothelial effect? Blood Cancer J 6:e384

119. Salem JE, Allenbach Y, Vozy A et al (2019) Abatacept for severe immune checkpoint inhibitor-associated myocarditis. N Engl J Med 380:2377-2379

120. Salem JE, Manouchehri A, Moey M et al (2018) Cardiovascular toxicities associated with immune checkpoint inhibitors: an observational, retrospective, pharmacovigilance study. Lancet Oncol 19:1579-1589

121. Sara JD, Kaur J, Khodadadi R et al (2018) 5-fluorouracil and cardiotoxicity: a review. Ther Adv Med Oncol 10:1758835918780140

122. Scherr M, Kirchhoff H, Battmer K et al (2019) Optimized induction of mitochondrial apoptosis for chemotherapy-free treatment of BCR-ABL+acute lymphoblastic leukemia. Leukemia 33:1313-1323

123. Shah MH, BinkleyP, Chan Ketal (2006) Cardiotoxicity of histone deacetylase inhibitor depsipeptide in patients with metastatic neuroendocrine tumors. Clin Cancer Res 12:3997-4003

124. Shah NP, Wallis N, Farber HW et al (2015) Clinical features of pulmonary arterial hypertension in patients receiving dasatinib. Am J Hematol 90:1060-1064

125. Siegel D, Martin T, Nooka A et al (2013) Integrated safety profile of single-agent carfilzomib: experience from 526 patients enrolled in 4 phase ll clinical studies. Haematologica 98:1753-1761

126. Slamon DJ, Leyland-Jones B, Shak S et al (2001) Use of chemotherapy plus a monoclonal antibody against HER2 for metastatic breast cancer that overexpresses HER2. NEngl J Med 344:783-792

127. Sorror ML, Giralt S, Sandmaier BM et al (2007) Hematopoieticcell transplantation specificcomor bidity index as an outcome predictor for patients with acute myeloid leukemia in first remission: combined FHCRC and MDACC experiences. Blood 110:4606-4613

128. Sorror ML, Maris MB, Storb R et al (2005) Hematopoietic cell transplantation (HCT)-specific comorbidity index: a new tool for risk assessment before allogeneic HCT. Blood 106:2912-2919

129. Steingart RM, Bakris GL, Chen HX et al (2012) Management of cardiac toxicity in patients receiving vascular endothelial growth factor signaling pathway inhibitors. Am Heart J 163:156-163

130. Steinherz LJ, Steinherz PG, Tan CT et al (1991) Cardiac toxicity 4 to 20 years after completing anthracycline therapy. JAMA 266:1672-1677

131. Stewart AK (2015) Carfilzomib for the treatment of patients with relapsed and/or refractory multiple myeloma. Future Oncol 11:2121-2136

132. Stocks T, Van Hemelrijck M, Manjer J et al (2012) Blood pressure and risk of cancer incidence and mortality in the metabolic syndrome and cancer project. Hypertension 59:802-810

133. Stuhlmiller TJ, Zawistowski JS, Chen $X$ et al (2017) Kinome and transcriptome profiling reveal broad and distinct activities of erlotinib, sunitinib and Sorafenib in the mouse heart and suggest cardiotoxicity from combined signal transducer and activator of transcription and epidermal growth factor receptor inhibition. J Am Heart Assoc. https://doi.org/10.1161/JAHA.117.006635

134. Subramanian S, Bates SE, Wright JJ et al (2010) Clinical toxicities of histone deacetylase inhibitors. Pharmaceuticals 3:2751-2767

135. Sun H, Li T, Zhuang R et al (2017) Do reninangiotensin system inhibitors influence the recurrence, metastasis, and survival in cancer patients?: Evidence from a meta-analysis including 55 studies. Medicine 96:e6394

136. Suzuki S, Ishikawa N, Konoeda F et al (2017) Nivolumab-related myasthenia gravis with myositis and myocarditis in Japan. Neurology 89:1127-1134

137. Swain SM, Whaley FS, Ewer MS (2003) Congestive heart failure in patients treated with doxorubicin: a retrospective analysis of three trials. Cancer 97:2869-2879

138. Taylor C, Correa C, Duane FK et al (2017) Estimating the risks of breast cancer radiotherapy: evidence from modern radiation doses to the lungs and heart and from previous randomized trials. J Clin Oncol 35:1641-1649

139. Terpos E, Kleber M, Engelhardt M et al (2015) European myeloma network guidelines for the management of multiple myeloma-related complications. Haematologica 100:1254-1266

140. Tilemann LM, Heckmann MB, Katus HA et a (2018) Cardio-oncology: conflicting priorities of anticancer treatment and cardiovascular outcome. Clin Res Cardiol 107:271-280

141. Totzeck M, Schuler M, Stuschke M et al (2019) Cardio-oncology_strategies for management of cancer-therapy related cardiovascular disease. Int J Cardiol 280:163-175

142. Trachtenberg BH, Landy DC, Franco VI et al (2011) Anthracycline-associated cardiotoxicity in survivors of childhood cancer. Pediatr Cardiol 32:342-353

143. Van Der PHJ, Van Dalen EC, Hauptmann M et al (2010) Cardiac function in 5-year survivors of childhood cancer: a long-term follow-up study. Arch Intern Med 170:1247-1255
144. Van Nimwegen FA, Schaapveld M, Janus CP et al (2015) Cardiovascular disease after Hodgkin lymphoma treatment: 40-year disease risk. JAMA Intern Med 175:1007-1017

145. Weatherald J, Chaumais MC, Savale L et al (2017) Long-term outcomes of dasatinib-induced pulmonary arterial hypertension: a populationbased study. Eur Respir J 50(1):1700217

146. Weberpals J, Jansen L, Muller OJ et al (2018) Longterm heart-specific mortality among 347476 breast cancer patients treated with radiotherapy or chemotherapy: a registry-based cohort study. Eur Heart J 39:3896-3903

147. Wiczer TE, Levine LB, Brumbaugh J et al (2017) Cumulative incidence, risk factors, and management of atrial fibrillation in patients receiving ibrutinib. Blood Adv 1:1739-1748

148. Yoon HJ, Kim KH, Kim HYetal (2019) Impacts ofnonrecovery of trastuzumab-induced cardiomyopathy on clinical outcomes in patients with breast cancer. Clin Res Cardiol 108:892-900

149. Zamorano JL, Lancellotti P, Rodriguez Munoz D et al (2016) 2016 ESC Position Paper on cancer treatments and cardiovascular toxicity developed under the auspices of the ESC Committee for Practice Guidelines: The Task Force for cancer treatments and cardiovascular toxicity of the European Society of Cardiology (ESC). Eur Heart J 37:2768-2801

150. Zhang Z, Huang TQ, Nepliouev I et al (2017) Crizotinib Inhibits Hyperpolarization-activated Cyclic Nucleotide-Gated Channel 4 Activity. Cardiooncology. https://doi.org/10.1186/s40959017-0020-z 